\title{
TWO-WEIGHT ESTIMATES FOR STRONG FRACTIONAL MAXIMAL FUNCTIONS AND POTENTIALS WITH MULTIPLE KERNELS
}

\author{
Vakhtang Kokilashvili and Alexander Meskhi
}

\begin{abstract}
In the paper two-weight inequalities of various type for strong fractional maximal functions and potentials with multiple kernels defined on $\mathbb{R}^{2}$ are established.
\end{abstract}

\section{Introduction}

In the present paper a full characterization of two-dimensional weight functions $v$ governing the trace inequality

$$
\left\|K_{\alpha, \beta} f\right\|_{L_{v}^{q}\left(\mathbb{R}^{2}\right)} \leq c\|f\|_{L^{p}\left(\mathbb{R}^{2}\right)},
$$

is given, where $K_{\alpha, \beta}$ is one of the following two-dimensional operators:

$$
\begin{aligned}
\left(M_{\alpha, \beta} f\right)(x, y) & =\sup _{I \times J \ni(x, y)} \frac{1}{|I|^{1-\alpha}|J|^{1-\beta}} \int_{I} \int_{J}|f(t, \tau)| d t d \tau ; \\
\left(I_{\alpha, \beta} f\right)(x, y) & =\int_{\mathbb{R}} \int_{\mathbb{R}}|x-t|^{\alpha-1}|y-\tau|^{\beta-1} f(t, \tau) d t d \tau ; \\
\left(I_{\alpha} J_{\beta} f\right)(x, y) & =\int_{\mathbb{R}} \int_{|\tau|<2|y|}|x-t|^{\alpha-1}|y-\tau|^{\beta-1} f(t, \tau) d t d \tau ; \\
\left(M_{\alpha} I_{\beta} f\right)(x, y) & =\sup _{I \ni x} \frac{1}{|I|^{1-\alpha}} \int_{I}\left|\int_{\mathbb{R}}\right| y-\left.\tau\right|^{\beta-1} f(t, \tau) d \tau \mid d t
\end{aligned}
$$

and $I$ and $J$ are arbitrary bounded intervals in $\mathbb{R}$ containing $x$ and $y$ respectively. For the operator $I_{\alpha, \beta}$ we additionally assume that the weight $v$ satisfies the Muckenhoupt one-dimensional $A_{\infty}(\mathbb{R})$ condition with respect to only one variable uniformly to another one. Moreover, criteria guaranteeing the twoweight inequality for these operators are obtained, provided that the weight

Received August 23, 2007.

2000 Mathematics Subject Classification. Primary 47G10, 26A33; Secondary 31B15, 42B25, 46E30.

Key words and phrases. strong fractional maximal functions, potentials with multiple kernels, two-weight inequality, trace inequality.

The work was partially supported by INTAS Grant No. 06-1000017-8792 and Georgian National Science Foundation Grant No. GNSF/ST06/3-010. 
on the right-hand side is a product of two one-dimensional weights. Dual inequalities of Fefferman-Stein type for strong fractional maximal functions and potentials with product kernels are also established.

The one-weight problem for the operator $I_{\alpha, \beta}$ has been solved in [22]. From the historical point of view we recall that the one-weight criteria for the Riesz potentials

$$
I_{\alpha} f(x)=\int_{\mathbb{R}^{n}} \frac{f(y)}{|x-y|^{n-\alpha}} d y, \quad 0<\alpha<n,
$$

have been derived in [34]. The pioneering results concerning the two-weight problem for $I_{\alpha}$ have been obtained in [40,41], while $L^{p}-L^{q}$ two-weight criteria in more transparent form have been given in $[14,15]$ for $1<p<q<\infty$ (see also $[17,42]$ regarding two-weight criteria for integral transforms with positive kernels). Namely, the next statement holds.

Theorem A. Let $1<p<q<\infty$ and let $0<\alpha<n$. Then $I_{\alpha}$ is bounded from $L_{w}^{p}\left(\mathbb{R}^{n}\right)$ into $L_{v}^{q}\left(\mathbb{R}^{n}\right)$ if and only if

$$
A_{1}:=\sup _{x \in \mathbb{R}^{n} ; r>0}\left(\int_{B(x, 2 r)} v\right)^{1 / q}\left(\int_{|x-y|>r}|x-y|^{(\alpha-n) p^{\prime}} w^{1-p^{\prime}}(y) d y\right)^{1 / p^{\prime}}<\infty
$$

and

$$
A_{2}:=\sup _{x \in \mathbb{R}^{n} ; r>0}\left(\int_{B(x, 2 r)} w^{1-p^{\prime}}\right)^{1 / p^{\prime}}\left(\int_{|x-y|>r}|x-y|^{(\alpha-n) q} v(y) d y\right)^{1 / q}<\infty,
$$

where $p^{\prime}=p /(p-1)$ and $B(x, r)$ is a ball with center $x$ and radius $r$. Moreover, there exist positive constants $c_{1}$ and $c_{2}$ depending only on $p, q$ and $\alpha$ such that

$$
c_{1} \max \left\{A_{1}, A_{2}\right\} \leq\left\|I_{\alpha}\right\| \leq c_{2} \max \left\{A_{1}, A_{2}\right\} .
$$

The proof of Theorem A is based on the two-weight weak-type criterion for the Riesz potentials given in [38] and on more transparent one established in $[13,15]$ (see also [25]). These criteria avoid the concept of capacity and can be easily verified.

Earlier, a capacitary characterization of those measures which guarantee the trace inequality for the operator $I_{\alpha}$ was derived in $[3,30]$.

In the case $w \equiv 1$, Theorem $\mathrm{A}$ (the trace inequality) has been obtained in [1].

Theorem A'. Let $1<p<q<\infty, 0<\alpha<n / p$. Then the operator $I_{\alpha}$ is bounded from $L^{p}\left(\mathbb{R}^{n}\right)$ to $L_{v}^{q}\left(\mathbb{R}^{n}\right)$ if and only if

$$
A^{\prime} \equiv \sup _{x \in \mathbb{R}^{n} ; r>0}\left(\int_{B(x, r)} v(x) d x\right) r^{q(\alpha-n / p)}<\infty .
$$

For $p=q$ the following two-weight criterion of pointwise type is due to [31] (see also [45] for more general case). 
Theorem B. Let $1<p<\infty, 0<\alpha<1 / p$. Then the operator $I_{\alpha}$ is bounded from $L^{p}\left(\mathbb{R}^{n}\right)$ to $L_{v}^{p}\left(\mathbb{R}^{n}\right)$ if and only if $I_{\alpha} v \in L_{l o c}^{p^{\prime}}\left(\mathbb{R}^{n}\right)$ and

$$
A_{3} \equiv \operatorname{essip}_{x \in \mathbb{R}^{n}}\left(\frac{I_{\alpha}\left[I_{\alpha} v\right]^{p^{\prime}}(x)}{I_{\alpha} v(x)}\right)^{1 / p^{\prime}}<\infty .
$$

Moreover,

$$
c_{1} A_{3} \leq\left\|I_{\alpha}\right\| \leq c_{2} A_{3},
$$

where the constants $c_{1}$ and $c_{2}$ depend only on $p$ and $\alpha$.

For the solution of the two-weight problem for fractional maximal operators

$$
M_{\alpha} f(x)=\sup _{B \ni x} \frac{1}{|B|^{1-\alpha / n}} \int_{B}|f|, \quad 0<\alpha<n,
$$

where the supremum is taken over all balls $B$ in $\mathbb{R}^{n}$ containing $x$, we refer to $[18,36,46]$ (see also [17]). A two-weight criterion for the strong HardyLittlewood maximal functions has been obtained in [36], provided that the weight on the right-hand side satisfies some additional conditions, for instance, belongs to the Muckenhoupt $A_{p}$ class in each variable separately, or is a product of one-dimensional weights. For some two-weight inequalities for fractional integrals and fractional maximal functions associated to starlike sets in $\mathbb{R}^{n}$ we refer to [6].

Necessary and sufficient conditions guaranteeing the trace inequalities for one-sided potentials with multiple kernels have been given in [26, 28, 29]. For some properties of potentials with product kernels in unweighted case see, e.g., [35, Ch. 5].

Two-weight inequalities for potential type operators can be applied, for example, to establish two-weight estimates for multipliers of Fourier transforms (see, e.g., [9]).

The paper is organized as follows: In Section 1 we present some well-known results concerning Carleson-Hörmander type inequality; doubling measures; two-weight estimates for the Hardy transforms and the truncated potentials. In Section 2 we formulate the main results of the paper, while in Section 3 we prove them.

Constants (often different constants in the same series of inequalities) will generally be denoted by $c$. For the real line and the set of all integers we will use the symbols $\mathbb{R}$ and $\mathbb{Z}$ respectively. The Lebesgue measure of the measurable set $E \subset \mathbb{R}^{n}$ will be denoted by $|E|$. We will also use the notation $\rho(E):=\int_{E} \rho$ for the weight $\rho$ on $\mathbb{R}^{n}$ and measurable set $E \subset \mathbb{R}^{n}$. For a set $I \subset \mathbb{R}$ and $t \in \mathbb{R}$, $I-t$ denotes the set $\{x-t: x \in I\}$.

Finally we mention that some of the results presented in this paper have been announced in $[27,29]$. 


\section{Preliminaries}

Let $\rho$ be an almost everywhere positive function on $\mathbb{R}^{n}$. By the symbol $L_{\rho}^{p}\left(\mathbb{R}^{n}\right)(1<p<\infty)$ we denote the weighted Lebesgue space which is the set of all measurable functions $f: \mathbb{R}^{n} \rightarrow \mathbb{R}$ for which

$$
\|f\|_{L_{\rho}^{p}\left(\mathbb{R}^{n}\right)}=\left(\int_{\mathbb{R}^{n}}|f(x)|^{p} \rho(x) d x\right)^{1 / p}<\infty .
$$

We shall need the following two-weight criterion for the Hardy operator

$$
H f(x)=\int_{|t|<|x|} f(t) d t, \quad x \in \mathbb{R},
$$

(see [4, 24, 33], [30, Ch. 1]).

Theorem C. Let $1<p \leq q<\infty$. Then $H$ is bounded from $L_{u}^{p}(\mathbb{R})$ to $L_{\sigma}^{q}(\mathbb{R})$ if and only if

$$
A_{4} \equiv \sup _{t>0}\left(\int_{|x|>t} \sigma(x) d x\right)^{1 / q}\left(\int_{|x|<t} u^{1-p^{\prime}}(x) d x\right)^{1 / p^{\prime}}<\infty .
$$

Moreover, there exist positive constants $c_{1}$ and $c_{2}$ depending only on $p$ and $q$ such that $c_{1} A_{4} \leq\|H\| \leq c_{2} A_{4}$.

The next statement concerning the truncated Riesz potential

$$
J_{\alpha} f(x)=\int_{|y|<2|x|} \frac{f(y)}{|x-y|^{n-\alpha}} d y, \quad x \in \mathbb{R}^{n},
$$

is due to [39] for $p=q$ (for the simple proof in the case $1<p \leq q<\infty$ see [10, Section 5.1]).

Theorem D. Let $1<p \leq q<\infty$. Suppose that $\alpha>n / p$. Then the operator $J_{\alpha}$ is bounded from $L^{p}\left(\mathbb{R}^{n}\right)$ to $L_{v}^{q}\left(\mathbb{R}^{n}\right)$ if and only if

$$
A_{5} \equiv \sup _{t>0}\left(\int_{|x|>t} \frac{v(x)}{|x|^{(n-\alpha) p}} d x\right)^{1 / q} t^{n / p^{\prime}}<\infty .
$$

Moreover, there exist positive constants $c_{1}$ and $c_{2}$ depending only on $p$ and $q$ such that

$$
c_{1} A_{5} \leq\left\|J_{\alpha}\right\| \leq c_{2} A_{5} .
$$

Let $\mathcal{D}$ be the set of all dyadic intervals in $\mathbb{R}$. By dyadic interval we mean an interval of the form $\left[2^{k} n, 2^{k}(n+1)\right)$, where $k$ and $n$ are integers. The main property of the dyadic intervals is that if $\left|I^{\prime}\right| \leq|I|$, then $I^{\prime} \subset I$ or $I^{\prime} \cap I=\emptyset$. Let us denote $\Lambda_{k}=2^{-k} \mathbb{Z}$ for $k \in \mathbb{Z}$. Suppose that $\mathcal{D}^{(k)}$ is the collection of the intervals determined by $\Lambda_{k}$. It is clear that $\mathcal{D}=\cup_{k \in \mathbb{Z}} \mathcal{D}^{(k)}$. Each $I \in \mathcal{D}^{(k)}$ is the union of 2 non-overlapping intervals belonging to $\mathcal{D}^{(k+1)}$ (for details and some properties of the dyadic intervals see, for instance, $[16$, p. 136]). 
Definition 1.1. We say that the weight function $\rho$ satisfies the dyadic reverse doubling condition $\left(\rho \in R D^{(d)}(\mathbb{R})\right)$ if there exists a constant $d>1$ such that

$$
d \rho\left(I^{\prime}\right) \leq \rho(I)
$$

for all $I^{\prime}, I \in \mathcal{D}$, where $I^{\prime} \subset I$ and $|I|=2\left|I^{\prime}\right|$.

It is obvious that the constant $d$ in Definition 1.1 is equal to 2 when $\rho \equiv$ 1. It is also easy to see that if a measure $\mu$ satisfies the doubling condition $\mu([x-2 r, x+2 r]) \leq b \mu([x-r, x+r])$ (i.e., $\mu \in D C(\mathbb{R}))$, where the constant $b$ is independent of $x \in \mathbb{R}$ and $r>0$, then $\mu \in D C^{(d)}(\mathbb{R})$, i.e., $\mu(I) \leq b_{1} \mu\left(I^{\prime}\right)$, where $I, I^{\prime} \in \mathcal{D}, I^{\prime} \subset I$ and $\left|I^{\prime}\right|=|I| / 2$. Consequently (see, e.g., [43, p. 21]) if $\mu \in D C(\mathbb{R})$, then $\mu \in R D^{(d)}(\mathbb{R})$.

We shall also need the next Carleson-Hörmander $([5,20])$ type embedding theorem regarding dyadic intervals (see $[41,44]$ ).

Theorem E. Let $1<p<q<\infty$ and let $\rho$ be a weight function on $\mathbb{R}$ such that $\rho^{1-p^{\prime}}$ satisfies the dyadic reverse doubling condition. Let $\left\{c_{I}\right\}$ be nonnegative numbers corresponding to dyadic intervals $I$ in $\mathbb{R}$. Then the following two statements are equivalent:

(i) There is a positive constant $C$ such that

$$
\sum_{I \in \mathcal{D}} c_{I}\left(\frac{1}{|I|} \int_{I} g(x) d x\right)^{q} \leq C\left(\int_{\mathbb{R}} g(x)^{p} \rho(x) d x\right)^{q / p}
$$

for all non-negative $g \in L_{\rho}^{p}(\mathbb{R})$;

(ii) There is a positive constant $C_{1}$ such that

$$
c_{I} \leq C_{1}|I|^{q}\left(\int_{I} \rho(x)^{1-p^{\prime}} d x\right)^{-q / p^{\prime}}
$$

for all $I \in \mathcal{D}$.

This result yields the following corollary.

Corollary A. Let $1<p<q<\infty$ and let $\rho$ be a weight function on $\mathbb{R}$ such that $\rho^{1-p^{\prime}}$ satisfies the dyadic reverse doubling condition. Then the CarlesonHörmander inequality

$$
\sum_{I \in \mathcal{D}}\left(\int_{I} \rho^{1-p^{\prime}}(x) d x\right)^{-q / p^{\prime}}\left(\int_{I} f(x) d x\right)^{q} \leq c\left(\int_{\mathbb{R}} f^{p}(x) \rho(x) d x\right)^{q / p}
$$

holds for all non-negative $f \in L_{\rho}^{p}(\mathbb{R})$.

\section{Main results}

Here we formulate the main results of this paper. Let us begin with the operator $M_{\alpha} I_{\beta}$. 
Theorem 2.1. Let $1<p<q<\infty$ and let $0<\alpha, \beta<1$. Suppose that $w(x, y)=w_{1}(x) w_{2}(y)$ with $w_{1}^{1-p^{\prime}} \in R D^{(d)}(\mathbb{R})$. Then $M_{\alpha} I_{\beta}$ is bounded from $L_{w}^{p}\left(\mathbb{R}^{2}\right)$ to $L_{v}^{q}\left(\mathbb{R}^{2}\right)$ if and only if

$$
\begin{aligned}
B_{1}:= & \sup _{a \in \mathbb{R} ; r>0 ; I \subset \mathbb{R}}|I|^{\alpha-1}\left(\int_{I} \int_{|y-a|<r} w^{1-p^{\prime}}(x, y) d x d y\right)^{1 / p^{\prime}} \\
& \times\left(\int_{I} \int_{|y-a|>r} \frac{v(x, y)}{|y-a|^{(1-\beta) q}} d x d y\right)^{1 / q}<\infty \\
B_{2}:= & \sup _{a \in \mathbb{R} ; r>0 ; I \subset \mathbb{R}}|I|^{\alpha-1}\left(\int_{I} \int_{|y-a|>r} w^{1-p^{\prime}}(x, y)|y-a|^{(\beta-1) p^{\prime}} d x d y\right)^{1 / p^{\prime}} \\
& \times\left(\int_{I} \int_{|y-a|<r} v(x, y) d x d y\right)^{1 / q}<\infty
\end{aligned}
$$

where $I$ is arbitrary bounded interval in $\mathbb{R}$.

Notice that $B_{1}$ is a dual of $B_{2}$.

For the strong fractional maximal operator we have:

Theorem 2.2. Let $1<p<q<\infty$ and let $0<\alpha, \beta<1$. Suppose that $w(x, y)=w_{1}(x) w_{2}(y)$ with $w_{1}^{1-p^{\prime}}, w_{2}^{1-p^{\prime}} \in R D^{(d)}(\mathbb{R})$. Then $M_{\alpha, \beta}$ is bounded from $L_{w}^{p}\left(\mathbb{R}^{2}\right)$ to $L_{v}^{q}\left(\mathbb{R}^{2}\right)$ if and only if

$$
B_{3}:=\sup _{I, J}|I|^{\alpha-1}|J|^{\beta-1}\left(\int_{I} \int_{J} v(x, y) d x d y\right)^{1 / q}\left(\int_{I} \int_{J} w^{1-p^{\prime}}(x, y) d x d y\right)^{1 / p^{\prime}}<\infty
$$

where the supremum are taken over all arbitrary bounded intervals $I$ and $J$ in $\mathbb{R}$.

To formulate the next result we need:

Definition 2.3. We say that the weight $\rho$ on $\mathbb{R}$ satisfies $A_{\infty}(\mathbb{R})$ condition $\left(\rho \in A_{\infty}(\mathbb{R})\right)$ if there exist constants $c, \delta>0$ such that for all intervals $I$ and measurable sets $E \subset I$ the inequality

$$
\frac{\rho(E)}{\rho(I)} \leq c\left(\frac{|E|}{|I|}\right)^{\delta}
$$

holds, where $\rho(E)=\int_{E} \rho$. Further, we say that a two-dimensional weight $u$ belongs to the class $A_{\infty}(\mathbb{R})$ with respect to the first variable uniformly to the second one $\left(u \in A_{\infty}^{(x)}(\mathbb{R})\right)$ if the inequality

$$
\frac{u_{y}(E)}{u_{y}(I)} \leq c\left(\frac{|E|}{|I|}\right)^{\delta}
$$

holds for all $y \in \mathbb{R}$, all intervals $I \subset \mathbb{R}$ and measurable sets $E \subset I$, where $u_{y}(E)=\int_{E} u(x, y) d x$. The class $A_{\infty}^{(y)}(\mathbb{R})$ is defined analogously. 
It is known (see [7, 21], [16, Ch. IV]) that $\rho \in A_{\infty}(\mathbb{R})$ if and only if $\rho$ belongs to the Muckenhoupt $A_{p}(\mathbb{R})$ class for some $p \geq 1$. It should be mentioned that some essential properties of the Muckenhoupt $A_{p}$ classes defined on rectangles has been studied in [11, 23] (see also [8], [16, Ch. IV]).

Our result concerning the Riesz potential with product kernels is:

Theorem 2.4. Let $1<p<q<\infty$ and let $0<\alpha, \beta<1$. Suppose that $w(x, y)=w_{1}(x) w_{2}(y)$ with $w_{1}^{1-p^{\prime}} \in R D^{(d)}(\mathbb{R})$ and $v \in A_{\infty}^{(x)}(\mathbb{R})$ uniformly to the second variable. Then $I_{\alpha, \beta}$ is bounded from $L_{w}^{p}\left(\mathbb{R}^{2}\right)$ to $L_{v}^{q}\left(\mathbb{R}^{2}\right)$ if and only if $\max \left\{B_{1}, B_{2}\right\}<\infty$.

Remark 2.5. Criteria for the boundedness of $I_{\alpha, \beta}$ from $L_{w}^{p}\left(\mathbb{R}^{2}\right)$ to $L_{v}^{p}\left(\mathbb{R}^{2}\right)$ provided that the weights $v$ and $w^{1-p}$ raised to a certain power satisfy $A_{\infty}$ condition uniformly with respect to the second variable have been announced in [29].

From Theorems 2.1 and 2.2 we have:

Corollary 2.6. Let $1<p<q<\infty$ and let $0<\alpha, \beta<1 / p$. Then the following statements are equivalent:

(i) $M_{\alpha} I_{\beta}$ is bounded from $L^{p}\left(\mathbb{R}^{2}\right)$ to $L_{v}^{q}\left(\mathbb{R}^{2}\right)$;

(ii) $M_{\alpha, \beta}$ is bounded from $L^{p}\left(\mathbb{R}^{2}\right)$ to $L_{v}^{q}\left(\mathbb{R}^{2}\right)$;

(iii)

$$
B_{4}:=\sup _{I, J}\left(\int_{I} \int_{J} v(x, y) d x d y\right)|I|^{q(\alpha-1 / p)}|J|^{q(\beta-1 / p)}<\infty,
$$

where $I$ and $J$ are arbitrary bounded intervals in $\mathbb{R}$.

Notice that in Corollary 2.6 there is no any additional condition on $v$ except (iii).

Theorem 2.4 yields:

Corollary 2.7. Let $1<p<q<\infty, 0<\alpha, \beta<1 / p$. Suppose that the twodimensional weight $v(x, y)$ belongs to $A_{\infty}^{(x)}(\mathbb{R})$ uniformly to $y$, or $v \in A_{\infty}^{(y)}(\mathbb{R})$ uniformly to $x$. Then the following statements are equivalent:

(i) $I_{\alpha, \beta}$ is bounded from $L^{p}\left(\mathbb{R}^{2}\right)$ to $L_{v}^{q}\left(\mathbb{R}^{2}\right)$;

(ii) $B_{4}<\infty$.

For the operator $I_{\alpha} J_{\beta}$ we have:

Theorem 2.8. Let $1<p<q<\infty$. Suppose that $0<\alpha<1$ and $\beta>1 / p$. Then the two-weight inequality

$$
\left(\iint_{\mathbb{R}^{2}}\left|\left(I_{\alpha} J_{\beta} f\right)(x, y)\right|^{q} v(x, y) d x d y\right)^{1 / q} \leq c\left(\iint_{\mathbb{R}^{2}}|f(x, y)|^{p} u(x) d x d y\right)^{1 / p}
$$

holds if and only if

$$
\text { (i) } \quad B_{5} \equiv \sup _{a \in \mathbb{R} ; r>0 ; k \in \mathbb{Z}}\left(\int_{|x-a|>r} \int_{2^{k}<|y|<2^{k+1}} \frac{v(x, y)}{|x-a|^{(1-\alpha) q}} d x d y\right)^{1 / q}
$$




$$
\times\left(\int_{|x-a|<r} u^{1-p^{\prime}}(x) d x\right)^{1 / p^{\prime}} 2^{k(\beta-1 / p)}<\infty
$$

$$
\begin{aligned}
B_{6} \equiv \sup _{a \in \mathbb{R} ; r>0 ; k \in \mathbb{Z}}\left(\int_{|x-a|<r} \int_{2^{k}<|y|<2^{k+1}} v(x, y) d x d y\right)^{1 / q} \\
\quad \times\left(\int_{|x-a|>r} \frac{u^{1-p^{\prime}}(x)}{|x-a|^{(\alpha-1) p^{\prime}}} d x\right)^{1 / p^{\prime}} 2^{k(\beta-1 / p)}<\infty .
\end{aligned}
$$

Corollary 2.9. Let $1<p<q<\infty$. Suppose that $0<\alpha<1$ and $\beta>1 / p$. Then the operator $I_{\alpha} J_{\beta}$ is bounded from $L^{p}\left(\mathbb{R}^{2}\right)$ to $L_{v}^{q}\left(\mathbb{R}^{2}\right)$ if and only if

$$
\sup _{a \in \mathbb{R} ; r>0 ; k \in \mathbb{Z}}\left(\int_{a-r}^{a+r} \int_{2^{k}<|y|<2^{k+1}} v(x, y) d x d y\right)^{1 / q} r^{\alpha-1 / p} 2^{k(\beta-1 / p)}<\infty .
$$

In the diagonal case $p=q$ we have:

Theorem 2.10. Let $1<p<\infty$. Suppose that $0<\alpha<1 / p<\beta$. Then the operator $I_{\alpha} J_{\beta}$ is bounded from $L^{p}\left(\mathbb{R}^{2}\right)$ to $L_{v}^{p}\left(\mathbb{R}^{2}\right)$ if and only if $I_{\alpha} \mathcal{V}_{j} \in L_{\text {loc }}^{p^{\prime}}(\mathbb{R})$ for all $j \in \mathbb{Z}$ and there exists a positive constant $c$ such that for almost all $x \in \mathbb{R}$ and all $j \in \mathbb{Z}$ the inequality

$$
I_{\alpha}\left[I_{\alpha} \mathcal{V}_{j}\right]^{p^{\prime}}(x) \leq c I_{\alpha}\left[\mathcal{V}_{j}\right](x)
$$

holds, where $I_{\alpha}$ is the one-dimensional potential and

$$
\mathcal{V}_{j}(x) \equiv \int_{2^{j}<|y|<2^{j+1}} v(x, y)|y|^{\beta p-1} d y .
$$

We have also the Fefferman-Stein type dual inequality for the operators $M_{\alpha, \beta}, M_{\alpha} I_{\beta}$ and $I_{\alpha, \beta}$. Notice that this inequality for the classical Riesz potentials $I_{\alpha}$ in the case $p=q$ was established by E. Sawyer (see, e.g., [2]).

Theorem 2.11. Let $1<p<q<\infty$. Suppose that $1 / p-1 / q<\alpha, \beta<1 / p$. Then there exists a positive constant $c$ such that

$$
\left(\iint_{\mathbb{R}^{2}}\left(M_{\alpha, \beta} f\right)^{q}(x, y) v(x, y) d x d y\right)^{1 / q} \leq c\left(\iint_{\mathbb{R}^{2}}|f(x, y)|^{p}\left(\widetilde{M}_{\alpha, \beta} v\right)^{p / q}(x, y) d x d y\right)^{1 / p},
$$

where

$$
\left(\widetilde{M}_{\alpha, \beta} v\right)(x, y):=\sup _{I \ni x ; J \ni y}|I|^{(\alpha-1 / p) q}|J|^{(\beta-1 / p) q} \int_{I} \int_{J} v(t, \tau) d t d \tau
$$

and the positive constant does not depend on $f$ and $v$.

Theorem 2.12. Let $1<p<q<\infty$ and let $1 / p-1 / q<\alpha, \beta<1 / p$. Suppose that $v \in A_{\infty}^{(y)}$ uniformly to $x$. Then the following inequality holds:

$$
\left(\iint_{\mathbb{R}^{2}}\left(M_{\alpha} I_{\beta} f\right)^{q}(x, y) v(x, y) d x d y\right)^{1 / q} \leq c\left(\iint_{\mathbb{R}^{2}}|f(x, y)|^{p}\left(\widetilde{M}_{\alpha, \beta} v\right)^{p / q}(x, y) d x d y\right)^{1 / p}
$$

with a positive constant $c$ independent of $f$. 
For $I_{\alpha, \beta}$ we have:

Theorem 2.13. Let $1<p<q<\infty$ and let $1 / p-1 / q<\alpha, \beta<1 / p$. Suppose that $v \in A_{\infty}^{(x)}(\mathbb{R}) \cap A_{\infty}^{(y)}(\mathbb{R})$. Then the following inequality

$\left(\iint_{\mathbb{R}^{2}}\left|\left(I_{\alpha, \beta} f\right)(x, y)\right|^{q} v(x, y) d x d y\right)^{1 / q} \leq c\left(\iint_{\mathbb{R}^{2}}|f(x, y)|^{p}\left(\widetilde{M}_{\alpha, \beta} v\right)^{p / q}(x, y) d x d y\right)^{1 / p}$ holds, where the positive constant $c$ does not depend on $f$.

\section{Proofs of the main results}

To prove the main statements of the paper we shall need the following lemma.

Lemma 3.1. Let $0<\alpha<1,0<\beta<1$. Suppose that $k \in \mathbb{Z}$ and $f \geq 0$. Then the following inequalities hold:

$$
\begin{gathered}
\left(M_{\alpha}^{\left(2^{k}\right)} I_{\beta}\right) f(x, y) \leq \frac{c_{\alpha}}{\left|R\left(0,2^{k+2}\right)\right|} \int_{R\left(0,2^{k+2}\right)} K_{t}(x, y) d t \\
M_{\alpha, \beta}^{\left(2^{k}\right)} f(x, y) \leq \frac{c_{\alpha, \beta}}{\left|R\left(0,2^{k+2}\right)\right|^{2}} \int_{R\left(0,2^{k+2}\right)} \int_{R\left(0,2^{k+2}\right)} S_{t, \tau}(x, y) d t d \tau,
\end{gathered}
$$

where

$$
\begin{aligned}
&\left(M_{\alpha}^{\left(2^{k}\right)} I_{\beta}\right) f(x, y):=\sup _{I \ni x ;|I| \leq 2^{k}} \frac{1}{|I|^{1-\alpha}} \int_{I} \int_{\mathbb{R}}|y-\tau|^{\beta-1} f(t, \tau) d t d \tau ; \\
& M_{\alpha, \beta}^{\left(2^{k}\right)} f(x, y):=\sup _{I \times J \ni(x, y) ;|I|,|J| \leq 2^{k}} \frac{1}{|I|^{1-\alpha}|J|^{1-\beta}} \int_{I} \int_{J} f(t, \tau) d t d \tau ; \\
& R(0, r)=\{t:-r \leq t \leq r\}, \quad r>0 ; \\
& K_{t}(x, y):=\sup _{I-t \ni x ; I \in \mathcal{D}} \frac{1}{|I|^{1-\alpha}} \int_{I-t}\left[I_{\beta} f(s, \cdot)\right](y) d s, \\
& I-t:=\{x-t: x \in I\} ; \sup _{I-t \ni x ; I \in \mathcal{D}} \sup _{J-\tau \ni y ; J \in \mathcal{D}} \frac{1}{|I|^{1-\alpha}} \frac{1}{|J|^{1-\beta}} \int_{I-t} \int_{J-\tau} f(s, \epsilon) d s d \epsilon .
\end{aligned}
$$

We notice that such type estimate first has been established in [12] for the classical Hardy-Littlewood maximal functions (see also [16, p. 431]. For fractional maximal functions see $[36,37])$.

Proof of Lemma 3.1. We shall need the following observation (see, e.g., [16, p. 431]). Let $j$ and $I$ be an integer and an interval respectively such that $2^{j-1}<|I| \leq 2^{j}$. Suppose that $k \in \mathbb{Z}, j \leq k$. Let $E$ be the set of those $t \in R\left(0,2^{k+2}\right)$ for which there is some $I_{1} \in \mathcal{D}-t$ with $\left|I_{1}\right|=2^{j+1}$ and such that $I \subset I_{1}$. Then

$$
|E| \geq 2^{k+2}
$$

where $\mathcal{D}-t:=\{I-t: I \in \mathcal{D}\}$. 
To prove (3.1) we take $f$ and $(x, y) \in \mathbb{R}^{2}$. Then there exists an interval $I$ $\left(|I| \leq 2^{k}\right)$ such that $x \in I$ and

$$
\frac{2}{|I|^{1-\alpha}} \int_{I}\left(I_{\beta} f(s, \cdot)\right)(y) d s \geq\left(M_{\alpha}^{\left(2^{k}\right)} I_{\beta}\right) f(x, y) .
$$

Let $j$ be an integer such that $2^{j-1}<|I| \leq 2^{j}$. In this case $j \leq k$. Let $E$ be the set defined above. Further, for every $t \in E$ there exists an interval $Q \in \mathcal{D}-t$ such that $|Q|=2^{j+1}, I \subset Q$ and

$$
\begin{aligned}
\frac{1}{2}\left(M_{\alpha}^{\left(2^{k}\right)} I_{\beta}\right) f(x, y) & \leq \frac{1}{|I|^{1-\alpha}} \int_{I}\left(I_{\beta} f(s, \cdot)\right)(y) d s \\
& \leq \frac{c}{|Q|^{1-\alpha}} \int_{Q}\left(I_{\beta} f(s, \cdot)\right)(y) d s \leq c K_{t}(x, y) .
\end{aligned}
$$

By (3.3) we also have that $|E| \geq\left|R\left(0,2^{k+2}\right)\right| / 2$. Hence

$$
\left(M_{\alpha}^{\left(2^{k}\right)} I_{\beta}\right) f(x, y) \leq \frac{c}{|E|} \int_{E} K_{t}(x, y) d t \leq \frac{c}{\left|R\left(0,2^{k+2}\right)\right|} \int_{R\left(0,2^{k+2}\right)} K_{t}(x, y) d t
$$

To prove (3.2) we argue as above. For given $f$ and $(x, y) \in \mathbb{R}^{2}$ we take intervals $R_{1} \ni x$ and $R_{2} \ni y,\left|R_{1}\right|,\left|R_{2}\right| \leq 2^{k}$, such that

$$
\frac{2}{\left|R_{1}\right|^{1-\alpha}\left|R_{2}\right|^{1-\beta}} \int_{R_{1}} \int_{R_{2}} f(s, \epsilon) d s d \epsilon>M_{\alpha, \beta}^{\left(2^{k}\right)} f(x, y) .
$$

Now, let us take integers $j$ and $i$ so that $2^{j-1}<\left|R_{1}\right| \leq 2^{j}$ and $2^{i-1}<\left|R_{2}\right| \leq 2^{i}$. It is obvious that $j, i \leq k$. Further, let us define the sets $E_{1}$ and $E_{2}$ by the following way:

$$
\begin{aligned}
& E_{1}:=\left\{t \in R\left(0,2^{k+2}\right): \exists I \in \mathcal{D}-t,|I|=2^{j+1}, R_{1} \subset I\right\} \\
& E_{2}:=\left\{\tau \in R\left(0,2^{k+2}\right): \exists J \in \mathcal{D}-\tau,|J|=2^{i+1}, R_{2} \subset J\right\} .
\end{aligned}
$$

Then for all $t \in E_{1}$ and $\tau \in E_{2}$ we have

$$
\frac{1}{2} M_{\alpha, \beta}^{\left(2^{k}\right)} f(x, y) \leq \frac{1}{\left|R_{1}\right|^{1-\alpha}\left|R_{2}\right|^{1-\beta}} \int_{R_{1}} \int_{R_{2}} f(s, \epsilon)(y) d s d \epsilon \leq c S_{t, \tau}(x, y) .
$$

By (3.3) we have that $\left|E_{1}\right|,\left|E_{2}\right| \geq\left|R\left(0,2^{k+2}\right)\right| / 2$. Hence

$$
\begin{aligned}
M_{\alpha, \beta}^{\left(2^{k}\right)} f(x, y) & \leq \frac{c}{\left|E_{1} \times E_{2}\right|} \int_{E_{1} \times E_{2}} S_{t, \tau}(x, y) d t d \tau \\
& \leq \frac{c}{\left|R\left(0,2^{k+2}\right)\right|^{2}} \int_{R\left(0,2^{k+2}\right)} \int_{R\left(0,2^{k+2}\right)} S_{t, \tau}(x, y) d t d \tau
\end{aligned}
$$

The lemma has been proved.

Proof of Theorem 2.1. Sufficiency. First let us show sufficiency for the dyadic version of the operator $M_{\alpha} I_{\beta}$ :

$$
N_{\alpha} I_{\beta} f(x, y)=\sup _{I \ni x ; I \in \mathcal{D}}|I|^{\alpha-1} \int_{I}\left|I_{\beta}(f(t, \cdot))(y)\right| d t .
$$


Let $f \geq 0$. Without loss of generality we can assume that $f$ is bounded and has a compact support. Therefore $N_{\alpha} I_{\beta}$ is finite for all $(x, y) \in \mathbb{R}^{2}$. We claim that if we prove the theorem for such functions, then by elementary discussion we can pass to arbitrary $f \in L_{w}^{p}\left(\mathbb{R}^{2}\right)$. Indeed, let $f \in L_{w}^{p}\left(\mathbb{R}^{2}\right)$ and let us take the sequence $f_{n}=f \chi_{B(0, n)} \chi_{\{f<n\}}$. Then $f_{n} \rightarrow f$ in $L_{w}^{p}\left(\mathbb{R}^{2}\right)$ and also pointwisely. Moreover, $f_{n}(x, y) \leq f(x, y)$. On the other hand, $\left(M_{\alpha} I_{\beta}\right) f_{n}$ is a Cauchy sequence, because

$\left\|\left(M_{\alpha} I_{\beta}\right) f_{n}-\left(M_{\alpha} I_{\beta}\right) f_{m}\right\|_{L_{v}^{q}\left(\mathbb{R}^{2}\right)} \leq\left\|\left(M_{\alpha} I_{\beta}\right)\left(f_{n}-f_{m}\right)\right\|_{L_{v}^{q}\left(\mathbb{R}^{2}\right)} \leq c\left\|f_{n}-f_{m}\right\|_{L_{w}^{p}\left(\mathbb{R}^{2}\right)}$.

Consequently, by the completeness of the space $L_{v}^{q}\left(\mathbb{R}^{2}\right)$ there exists $g \in L_{v}^{q}\left(\mathbb{R}^{2}\right)$ such that

$$
\left\|\left(M_{\alpha} I_{\beta}\right) f_{n}-g\right\|_{L_{v}^{q}\left(\mathbb{R}^{2}\right)} \rightarrow 0 .
$$

Hence, there exists a subsequence $\left(M_{\alpha} I_{\beta}\right) f_{n_{k}}$ which converges in norm and almost everywhere to $g$. But $f_{n_{k}}$ converges to $f$ in $L_{w}^{p}$ and also a.e.. This leads to the inequality

$$
\|g\|_{L_{v}^{q}\left(\mathbb{R}^{2}\right)} \leq c\|f\|_{L_{w}^{p}\left(\mathbb{R}^{2}\right)},
$$

where $c$ does not depend on $f$.

Now we will argue for the subsequence $f_{n_{k}}$ :

For all $(x, y) \in \mathbb{R}^{2}$ with $x \in I$ we have $\left(f_{n_{k}}\right.$ is non-decreasing)

$$
\begin{aligned}
|I|^{\alpha-1} \int_{I} \int_{\mathbb{R}} \frac{f(t, \tau)}{|y-\tau|^{1-\beta}} d t d \tau & =\lim _{k \rightarrow \infty}|I|^{\alpha-1} \int_{I} \int_{\mathbb{R}} \frac{f_{n_{k}}(t, \tau)}{|y-\tau|^{1-\beta}} d t d \tau \\
& \leq \lim _{k \rightarrow \infty} \sup _{I, I \ni x}|I|^{\alpha-1} \int_{I} \int_{\mathbb{R}} \frac{f_{n_{k}}(t, \tau)}{|y-\tau|^{1-\beta}} d t d \tau \\
& =\lim _{k \rightarrow \infty}\left(M_{\alpha} I_{\beta}\right) f_{n_{k}}(x, y)
\end{aligned}
$$

and the last limit exists, because it converges a.e. to $g$. Hence

$$
\left(M_{\alpha} I_{\beta}\right) f(x, y) \leq \lim _{k \rightarrow \infty}\left(M_{\alpha} I_{\beta}\right) f_{n_{k}}(x, y)=g(x, y)
$$

for almost all $(x, y)$. Finally, by $(3.4)$ we have

$$
\left\|\left(M_{\alpha} I_{\beta}\right) f\right\|_{L_{v}^{q}\left(\mathbb{R}^{2}\right)} \leq c\|f\|_{L_{w}^{p}\left(\mathbb{R}^{2}\right)} .
$$

Further, for $(x, y) \in \mathbb{R}^{2}$ there exists dyadic interval $I_{y}(x) \subset \mathbb{R}$ containing $x$ such that

$$
\frac{2}{\left|I_{y}(x)\right|^{1-\alpha}} \int_{I_{y}(x)} I_{\beta}(f(t, \cdot))(y) d t \geq N_{\alpha} I_{\beta} f(x, y) .
$$

Let

$$
F_{I}=\left\{(x, y) \in \mathbb{R}^{2}: x \in I \text { and } I \text { is minimal for which (3.5) holds }\right\} .
$$

Let $\mathcal{D}_{m}$ be a subset of $\mathcal{D}$ for which $F_{I} \neq \emptyset$. By the main property of dyadic intervals it is clear that $F_{I} \cap F_{J}=\emptyset$ when $I \neq J$. Notice also that from the 
inequality

$$
\left(N_{\alpha} I_{\beta}\right) f(x, y) \leq \frac{2}{\left|I_{y}(x)\right|^{1-\alpha}} \int_{I_{y}(x)} I_{\beta} f(t, \cdot)(y) d t \leq c\left|I_{y}(x)\right|^{\alpha},
$$

with the constant $c$ depending on $L^{\infty}$ norm and the support of $f$, it follows the existence of minimal dyadic interval containing $x$ for which (3.5) holds.

From the definition of maximal operator and the latter inequality it is obvious that $\mathbb{R}^{2}=\cup_{I \in \mathcal{D}_{m}} F_{I}$. It is also clear that $F_{I} \subset I \times \mathbb{R}$ and that $F_{I}$ is a measurable set for every $I$. Let us use the notation:

$$
V_{I}(y):=|I|^{(\alpha-1) q} \int_{I} v(x, y) d x ; \quad \bar{F}_{I}(\tau):=\int_{I} f(t, \tau) d t .
$$

We have

$$
\begin{aligned}
& \int_{\mathbb{R}} \int_{\mathbb{R}}\left[\left(N_{\alpha} I_{\beta}\right) f(x, y)\right]^{q} v(x, y) d x d y \\
\leq & 2^{q} \sum_{I \in \mathcal{D}_{m}} \iint_{F_{I}}\left[\frac{1}{|I|^{1-\alpha}} \int_{I} I_{\beta}(f(t, \cdot))(y) d t\right]^{q} v(x, y) d x d y \\
\leq & 2^{q} \sum_{I \in \mathcal{D}_{m}} \int_{\mathbb{R}} V_{I}(y)\left[\int_{I}\left(\int_{\mathbb{R}}|y-\tau|^{\beta-1} f(t, \tau) d \tau\right) d t\right]^{q} d y
\end{aligned}
$$

(change the order of integration in the inner integral)

$=2^{q} \sum_{I \in \mathcal{D}_{m}} \int_{\mathbb{R}} V_{I}(y)\left[\int_{\mathbb{R}}|y-\tau|^{\beta-1} \bar{F}_{I}(\tau) d \tau\right]^{q} d y$

(by Theorem $\mathrm{A}$ and the fact that in the case $n=1$ the first integrals in $A_{1}$ and $A_{2}$ of Theorem A can be taken over intervals $(x-r, x+r)$, see [14])

$$
\begin{aligned}
& \leq c\left(\max \left\{B_{1}, B_{2}\right\}\right)^{q} \sum_{I \in \mathcal{D}_{m}}\left[\int_{\mathbb{R}}\left(\int_{I} w_{1}^{1-p^{\prime}}(t) d t\right)^{1-p}\left(\bar{F}_{I}(y)\right)^{p} w_{2}(y) d y\right]^{q / p} \\
& =c \sum_{I \in \mathcal{D}_{m}}\left(\int_{I} w_{1}^{1-p^{\prime}}(t) d t\right)^{-q / p^{\prime}}\left[\int_{\mathbb{R}}\left(\bar{F}_{I}(y)\right)^{p} w_{2}(y) d y\right]^{q / p}
\end{aligned}
$$

(due to Minkowski's integral inequality and Corollary A)

$$
\leq \sum_{I \in \mathcal{D}_{m}}\left(\int_{I} w_{1}^{1-p^{\prime}}(t) d t\right)^{-q / p^{\prime}}\left[\int_{I}\left(\int_{\mathbb{R}} f^{p}(t, y) w_{2}(y) d y\right)^{1 / p} d t\right]^{q} \leq c\|f\|_{L_{w}^{p}\left(\mathbb{R}^{2}\right)}^{q} .
$$

Hence $N_{\alpha} I_{\beta}$ is bounded from $L_{w}^{p}\left(\mathbb{R}^{2}\right)$ to $L_{v}^{q}\left(\mathbb{R}^{2}\right)$.

Let us now pass to the operator $M_{\alpha} I_{\beta}$. Assume that $k \in \mathbb{Z}$. Due to Lemma 3.1 we have

$$
D_{t}:=\iint_{\mathbb{R}^{2}}\left(K_{t}(x, y)\right)^{q} v(x, y) d x d y
$$




$$
=\iint_{\mathbb{R}^{2}}\left(\sup _{x \in I ; I \in \mathcal{D}} \frac{1}{|I|^{1-\alpha}} \int_{I} \int_{\mathbb{R}}|y-\epsilon|^{\beta-1} f(s-t, \epsilon) d \epsilon d s\right)^{q} v(x-t, y) d x d y
$$

(by the condition $\max \left\{B_{1}, B_{2}\right\}<\infty$ and the fact that this condition is translation invariant)

$$
\begin{aligned}
& \leq c\left(\max \left\{B_{1}, B_{2}\right\}\right)^{q}\left(\iint_{\mathbb{R}^{2}}(f(x-t, y))^{p} w(x-t, y) d x d y\right)^{q / p} \\
& =c\left(\iint_{\mathbb{R}^{2}}(f(x, y))^{p} w(x, y) d x d y\right)^{q / p} .
\end{aligned}
$$

Consequently,

$$
\left(D_{t}\right)^{1 / q} \leq c\left(\iint_{\mathbb{R}^{2}}(f(x, y))^{p} w(x, y) d x d y\right)^{1 / p},
$$

where the constant $c$ does not depend on $t$.

Applying Lemma 3.1, Minkowski's inequality and the latter inequality we find that

$$
\begin{aligned}
& \left(\iint_{\mathbb{R}^{2}}\left[\left(M_{\alpha}^{\left(2^{k}\right)} I_{\beta}\right) f(x, y)\right]^{q} v(x, y) d x d y\right)^{1 / q} \\
\leq & c\left(\iint_{\mathbb{R}^{2}}\left(\frac{1}{\left|R\left(0,2^{k+2}\right)\right|} \int_{R\left(0,2^{k+2}\right)} K_{t}(x, y) d t\right)^{q} v(x, y) d x d y\right)^{1 / q} \\
\leq & c\left|R\left(0,2^{k+2}\right)\right|^{-1} \int_{R\left(0,2^{k+2}\right)}\left(\iint_{\mathbb{R}^{2}} K_{t}^{q}(x, y) v(x, y) d x d y\right)^{1 / q} d t \\
= & c\left|R\left(0,2^{k+2}\right)\right|^{-1} \iint_{R\left(0,2^{k+2}\right)}\left(D_{t}\right)^{1 / q} d t \leq c\left(\int_{\mathbb{R}^{2}}(f(x, y))^{p} w(x, y) d x d y\right)^{1 / p} .
\end{aligned}
$$

Passing now $k$ to the infinity we finally obtain sufficiency.

To prove necessity we take the functions

$$
f(x, y)=\chi_{I}(x) \chi_{\{y:|y-a|>r\}}(y) w^{1-p^{\prime}}(x, y)|a-y|^{\left(p^{\prime}-1\right)(\beta-1)},
$$

where $a \in \mathbb{R}$ and $I$ is an interval in $\mathbb{R}$. Then we have

$$
\begin{aligned}
& \iint_{\mathbb{R}^{2}} v(x, y)\left[\left(M_{\alpha} I_{\beta}\right) f(x, y)\right]^{q} d x d y \\
\geq & \int_{I} \int_{|y-a|<r} v(x, y)|I|^{(\alpha-1) q}\left(\int_{I} \int_{|\tau-a|>r} \frac{f(t, \tau)}{|y-\tau|^{1-\beta}} d t d \tau\right)^{q} d x d y \\
& (\text { if }|y-a|<r \text { and }|\tau-a|>r, \text { then }|\tau-y| \leq 2|\tau-a|) \\
\geq & c\left(\int_{I} \int_{|y-a|<r} v(x, y) d x d y\right)|I|^{(\alpha-1) q}\left(\int_{I} \int_{|\tau-a|>r} \frac{w^{1-p^{\prime}}(t, \tau)}{|\tau-a|^{(1-\beta) p^{\prime}}} d t d \tau\right)^{q} .
\end{aligned}
$$


On the other hand,

$$
\|f\|_{L_{w}^{p}\left(\mathbb{R}^{2}\right)}^{q}=\left(\int_{I} \int_{|y-a|>r} w^{1-p^{\prime}}(x, y)|y-a|^{(\beta-1) p^{\prime}} d x d y\right)^{q / p} .
$$

From the two-weight inequality we will derive the condition $B_{2}<\infty$. To prove $B_{1}<\infty$ we take the functions

$$
f(x, y)=\chi_{I}(x) \chi_{\{y:|y-a|<r\}}(y) w^{1-p^{\prime}}(x, y)
$$

and argue as above. Theorem 2.1 has been proved.

Proof of Theorem 2.2. First we prove the theorem for the operator

$$
N_{\alpha, \beta} f(x, y):=\sup _{I \times J \ni(x, y) ; I, J \in \mathcal{D}}|I|^{\alpha-1}|J|^{\beta-1} \int_{I} \int_{J}|f(t, \tau)| d t d \tau .
$$

We can assume that $f$ is a non-negative bounded function with compact support. In this case $N_{\alpha, \beta} f(x, y)<\infty$ for all $(x, y) \in \mathbb{R}^{2}$.

For every $(x, y) \in \mathbb{R}^{2}$ let us take the pair of dyadic intervals $\{I(x), J(y)\}$, $x \in I(x), y \in J(y)$ such that

$$
\frac{2}{|I(x)|^{1-\alpha}|J(y)|^{1-\beta}} \int_{I(x)} \int_{J(y)} f(t, \tau) d t d \tau \geq N_{\alpha, \beta} f(x, y) .
$$

Let us introduce the set

$$
F_{I, J}=\left\{(x, y) \in \mathbb{R}^{2}: x \in I, y \in J \text { and (3.6) holds for } I \text { and } J\right\}
$$

for each $(I, J), I, J \in \mathcal{D}$. In this case for a fixed point $(x, y)$ the minimal dyadic rectangle $I \times J$ satisfying (3.6) (i.e., for any proper dyadic rectangle $I_{1} \times J_{1}$, $I_{1} \subset I, J_{1} \subset J$ (3.6) fails) exists but the latter is not unique. For our reasons it is enough that (3.6) holds on every $(x, y) \in F_{I, J} ; \mathbb{R}^{2}=\cup_{I, J \in \mathcal{D}} F_{I, J}$ and $F_{I, J} \subset I \times J$.

In the sequel we will use the notation:

$$
\bar{V}_{I, J}:=|I|^{(\alpha-1) q}|J|^{(\beta-1) q} \int_{I} \int_{J} v(x, y) d x d y ; \quad \bar{F}_{I, J}:=\int_{I} \int_{J} f(t, \tau) d t d \tau .
$$

We have

$$
\begin{aligned}
& \iint_{\mathbb{R}^{2}}\left[N_{\alpha, \beta} f(x, y)\right]^{q} v(x, y) d x d y \\
\leq & 2^{q} \sum_{I, J \in \mathcal{D}} \bar{V}_{I, J} \bar{F}_{I, J}^{p}\left(\text { by the condition } B_{3}<\infty\right)
\end{aligned}
$$$$
\leq 2^{q} B_{3}^{q} \sum_{I, J \in \mathcal{D}}\left(\int_{I} w_{1}^{1-p^{\prime}}(t) d t\right)^{-q / p^{\prime}}\left(\int_{J} w_{2}^{1-p^{\prime}}(t) d t\right)^{-q / p^{\prime}}\left(\int_{I} \int_{J} f(t, \tau) d t d \tau\right)^{q}
$$$$
=c \sum_{J \in \mathcal{D}}\left(\int_{J} w_{2}^{1-p^{\prime}}(t) d t\right)^{-q / p^{\prime}} \sum_{I \in \mathcal{D}}\left(\int_{I} w_{1}^{1-p^{\prime}}(t) d t\right)^{-q / p^{\prime}}\left[\int_{I}\left(\int_{J} f(t, \tau) d \tau\right) d t\right]^{q}
$$

(due to Corollary A with respect to the intervals $I$ ) 
$\leq c \sum_{J \in \mathcal{D}}\left(\int_{J} w_{2}^{1-p^{\prime}}(t) d t\right)^{q / p}\left(\int_{\mathbb{R}} w_{1}(t)\left(\int_{J} f(t, \tau) d \tau\right)^{p} d t\right)^{q / p}$

(by generalized Minkowski's inequality and Corollary A)

$\leq c \sum_{J \in \mathcal{D}}\left(\int_{J} w_{2}^{1-p^{\prime}}(t) d t\right)^{q / p}\left(\int_{J}\left(\int_{\mathbb{R}} f^{p}(t, \tau) w_{1}(t) d t\right)^{1 / p} d \tau\right)^{q} \leq c\|f\|_{L_{w}^{p}\left(\mathbb{R}^{2}\right)}^{q}$.

Arguing in the same manner as in the proof of Theorem 2.1, using (3.2) and the fact that the condition $B_{3}<\infty$ is translation invariant, we can derive sufficiency of the theorem.

To show necessity we observe that

$$
\left\|M_{\alpha, \beta} f\right\|_{L_{v}^{q}\left(\mathbb{R}^{2}\right)} \geq|I|^{\alpha-1}|J|^{\beta-1}\left(\int_{I} \int_{J} v(x, y) d x d y\right)^{1 / q}\left(\int_{I} \int_{J} f(t, \tau) d t d \tau\right)
$$

for any pair of intervals $(I, J)$. It remains now to use the two-weight inequality for the functions

$$
f(x, y)=\chi_{I}(x) \chi_{J}(y) w^{1-p^{\prime}}(x, y) .
$$

The next statement is well-known ([34]).

Proposition 3.2. Let $1<q<\infty$. Suppose that $0<\alpha<1$ and $\rho \in A_{\infty}(\mathbb{R})$. Then there exists a positive constant $c$ depending only on $\alpha, p$ and $w$ such that the inequality

holds.

$$
\left\|I_{\alpha} f\right\|_{L_{\rho}^{q}(\mathbb{R})} \leq c\left\|M_{\alpha} f\right\|_{L_{\rho}^{q}(\mathbb{R})}
$$

Applying Proposition 3.2 to one of the variables uniformly with respect to the second one we easily derive Theorem 2.4.

Proof of Corollary 2.6. To prove the implication (iii) $\Rightarrow$ (i) observe that the condition $B_{4}<\infty$ implies $\max \left\{B_{1}, B_{2}\right\}<\infty$ for $w \equiv 1$. Indeed, we have

$$
B_{2}:=c \sup _{a \in \mathbb{R} ; r>0 ; I \subset \mathbb{R}}|I|^{\alpha-1 / p} r^{\beta-1 / p}\left(\int_{I} \int_{|y-a|<r} v(x, y) d x d y\right)^{1 / q}=c B_{4}^{1 / q},
$$

while

$$
B_{1}:=c \sup _{a \in \mathbb{R} ; r>0 ; I \subset \mathbb{R}}|I|^{\alpha-1 / p} r^{1 / p^{\prime}}\left(\int_{I} \int_{|y-a|>r} \frac{v(x, y)}{|y-a|^{(1-\beta) q}} d x d y\right)^{1 / q} .
$$

Further,

$$
\begin{aligned}
& |I|^{(\alpha-1 / p) q} r^{q / p^{\prime}} \int_{I} \int_{|y-a|>r} \frac{v(x, y)}{|y-a|^{(1-\beta) q}} d x d y \\
= & |I|^{(\alpha-1 / p) q} r^{q / p^{\prime}} \sum_{k=0}^{\infty} \int_{I} \int_{2^{k} r<|y-a|<2^{k+1} r} \frac{v(x, y)}{|y-a|^{(1-\beta) q}} d x d y
\end{aligned}
$$




$$
\begin{aligned}
& \leq|I|^{(\alpha-1 / p) q} r^{q / p^{\prime}} \sum_{k=0}^{\infty}\left(2^{k} r\right)^{(\beta-1) q} \int_{I} \int_{2^{k} r<|y-a|<2^{k+1} r} v(x, y) d x d y \\
& \leq c B_{4} r^{q / p^{\prime}} \sum_{k=0}^{\infty}\left(2^{k} r\right)^{-q / p^{\prime}}=c B_{4} \sum_{k=0}^{\infty} 2^{-k q / p^{\prime}}=c B_{4} .
\end{aligned}
$$

Hence, $B_{2} \leq c B_{4}^{1 / q}$ and consequently, $\max \left\{B_{1}, B_{2}\right\} \leq c B_{4}^{1 / q}$ when $w \equiv 1$. Now it remains to apply Theorem 2.1. It is easy to see that the implication (ii) $\Rightarrow$ (iii) follows from the boundedness of the operator $M_{\alpha, \beta}$ on the class of functions

$$
f_{I, J}(x, y)=\chi_{I}(x) \chi_{J}(y) .
$$

Due to the inequality $M_{\alpha, \beta} f(x, y) \leq M_{\alpha} I_{\beta} f(x, y)(f \geq 0)$ we find that (i) $\Rightarrow$ (ii). Finally we have (iii) $\Rightarrow$ (i) $\Rightarrow$ (ii) $\Rightarrow$ (iii).

Proof of Corollary 2.7. First we prove the implication (ii) $\Rightarrow$ (i). We have already shown (see the proof of Corollary 2.6) that $\max \left\{B_{1}, B_{2}\right\} \leq c B_{4}^{1 / q}$. Further, let $v \in A_{\infty}^{(x)}$. Then the results follows directly from Theorem 2.4. Let now $v \in A_{\infty}^{(y)}$. Then taking into account the previous case we have

$\iint_{\mathbb{R}^{2}} v(x, y)\left(I_{\alpha, \beta} f\right)^{q}(x, y) d x d y=\iint_{\mathbb{R}^{2}} v^{*}(y, x)\left(I_{\beta, \alpha} f_{1}\right)^{q}(y, x) d y d x \leq c\|f\|_{L^{p}\left(\mathbb{R}^{2}\right)}^{q}$, where $v^{*}(y, x)=v(x, y), f_{1}(\tau, t)=f(t, \tau)$. It remains to notice that the condition $v \in A_{\infty}^{(y)}$ is equivalent to the fact $v^{*} \in A_{\infty}^{(x)}$. The implication (ii) $\Rightarrow$ (i) has been proved.

The fact (i) $\Rightarrow$ (ii) follows from Corollary 2.6 using the obvious inequality $M_{\alpha, \beta} f(x, y) \leq I_{\alpha, \beta} f(x, y), f \geq 0$.

To prove Theorem 2.8 we need the next statement concerning the operator

$$
\left(I_{\alpha} H\right) f(x, y)=\int_{\mathbb{R}} \int_{|\tau|<|y|} \frac{f(t, \tau)}{|x-t|^{1-\alpha}} d t d \tau .
$$

Proposition 3.3. Let $1<p<q<\infty, 0<\alpha<1$. Suppose that $w(x, y)=$ $w_{1}(x) w_{2}(y)$. Then the operator $I_{\alpha} H$ is bounded from $L_{w}^{p}\left(\mathbb{R}^{2}\right)$ to $L_{v}^{q}\left(\mathbb{R}^{2}\right)$ if and only if

$$
\begin{aligned}
D_{1} \equiv & \sup _{a \in \mathbb{R} ; r>0 ; s>0}\left(\int_{|x-a|<r} \int_{|y|<s} w^{1-p^{\prime}}(x, y) d x d y\right)^{1 / p^{\prime}} \\
& \times\left(\int_{|x-a|>r} \int_{|y|>s} \frac{v(x, y)}{|x-a|^{(1-\alpha) q}} d x d y\right)^{1 / q}<\infty ;
\end{aligned}
$$

$$
\begin{aligned}
D_{2} \equiv & \sup _{a \in \mathbb{R} ; r>0 ; s>0}\left(\int_{|x-a|>r} \int_{|y|<s} \frac{w^{1-p^{\prime}}(x, y)}{|x-a|^{(1-\alpha) p^{\prime}}} d x d y\right)^{1 / p^{\prime}} \\
& \times\left(\int_{|x-a|<r} \int_{|y|>s} v(x, y) d x d y\right)^{1 / q}<\infty
\end{aligned}
$$


hold.

This statement in more general form has been formulated in [29].

To prove this proposition we need the following statements (see, e.g., [32]).

Lemma 3.4. Let $1<p \leq q<\infty$ and let $\left\{a_{n}\right\},\left\{b_{n}\right\}$ be positive sequences. The inequality

$$
\left(\sum_{n=-\infty}^{\infty}\left|\sum_{k=-\infty}^{n} g_{k}\right|^{q} a_{n}^{q}\right)^{1 / q} \leq c\left(\sum_{n=-\infty}^{\infty}\left|g_{n}\right|^{p} b_{n}^{p}\right)^{1 / p}
$$

with the positive constant $c$ independent of $\left\{g_{k}\right\}\left(\left\{g_{k}\right\} \in l_{b_{n}^{p}}^{p}(\mathbb{Z})\right)$, holds if and only if

$$
\bar{B}:=\sup _{n \in \mathbb{Z}}\left(\sum_{k=n}^{\infty} a_{k}^{q}\right)^{1 / q}\left(\sum_{k=-\infty}^{n} b_{k}^{-p^{\prime}}\right)^{1 / p^{\prime}}<\infty .
$$

Moreover, if $c$ is the best constant in (3.7), then

$$
\bar{B} \leq c \leq \bar{B} q^{\frac{1}{q}}\left(\frac{q}{q-1}\right)^{(p-1) / p} .
$$

Lemma 3.5. Let $1<p \leq q<\infty$ and let $m$ be an integer. Suppose that $\left\{a_{n}\right\}_{n=-\infty}^{m},\left\{b_{n}\right\}_{n=-\infty}^{m}$ are positive sequences. Then the two-weight inequality

$$
\left(\sum_{n=-\infty}^{m}\left|\sum_{k=-\infty}^{n} g_{k}\right|^{q} a_{n}^{q}\right)^{1 / q} \leq c\left(\sum_{n=-\infty}^{m}\left|g_{n}\right|^{p} b_{n}^{p}\right)^{1 / p}
$$

for all $\left\{g_{k}\right\}_{k=-\infty}^{m}$ holds if and only if

$$
B^{(m)}:=\sup _{-\infty<n \leq m}\left(\sum_{k=n}^{m} a_{k}^{q}\right)^{1 / q}\left(\sum_{k=-\infty}^{n} b_{k}^{-p^{\prime}}\right)^{1 / p^{\prime}}<\infty .
$$

Moreover, if $c$ is the best constant in (3.8), then

$$
B^{(m)} \leq c \leq B^{(m)} q^{\frac{1}{q}}\left(\frac{q}{q-1}\right)^{(p-1) / p} .
$$

Proof of Proposition 3.3. Sufficiency. First suppose that $S:=\int_{\mathbb{R}} w_{2}^{1-p^{\prime}}(y) d y=$ $\infty$.

Let $\left\{x_{k}\right\}_{k=-\infty}^{+\infty}$ be a sequence of positive numbers for which the equality

$$
2^{k}=\int_{|y|<x_{k}} w_{2}^{1-p^{\prime}}(y) d y
$$

holds for all $k \in \mathbb{Z}$. It is clear that $\left\{x_{k}\right\}$ is increasing and

$$
\mathbb{R} \backslash\{0\}=\cup_{k \in \mathbb{Z}} E_{k},
$$

where $E_{k}:=\left\{y \in \mathbb{R}: x_{k} \leq|y|<x_{k+1}\right\}$. Besides, it is easy to verify that $2^{k}=\int_{E_{k}} w_{2}^{1-p^{\prime}}$. Let $f \geq 0$. We have

$$
I:=\int_{\mathbb{R}} \int_{\mathbb{R}} v(x, y)\left(I_{\alpha} H f(x, y)\right)^{q} d x d y
$$




$$
\begin{aligned}
& =\sum_{k \in \mathbb{Z}} \int_{\mathbb{R}} \int_{E_{k}} v(x, y)\left(\int_{\mathbb{R}} \int_{|\tau|<|y|} \frac{f(t, \tau)}{|x-t|^{1-\alpha}} d t d \tau\right)^{q} d x d y \\
& \leq \sum_{k \in \mathbb{Z}} \int_{\mathbb{R}}\left(\int_{E_{k}} v(x, y) d y\right)\left(\int_{\mathbb{R}}\left(\int_{|\tau|<x_{k+1}} \frac{f(t, \tau)}{|x-t|^{1-\alpha}} d \tau\right) d t\right)^{q} d x \\
& =\sum_{k \in \mathbb{Z}} \int_{\mathbb{R}} \bar{v}_{k}(x)\left(\int_{\mathbb{R}}|x-t|^{\alpha-1} F_{k}(t) d t\right)^{q} d x
\end{aligned}
$$

where

$$
\bar{v}_{k}(x):=\int_{E_{k}} v(x, y) d y ; \quad F_{k}(t):=\int_{|\tau|<x_{k+1}} f(t, \tau) d \tau .
$$

Further, by Theorem A,

$$
\begin{aligned}
I & \leq c\left(\max \left\{D_{1}, D_{2}\right\}\right)^{q} \sum_{j \in \mathbb{Z}}\left[\int_{\mathbb{R}} w_{1}(x)\left(\int_{|y|<x_{j}} w_{2}^{1-p^{\prime}}(y) d y\right)^{1-p}\left(F_{k}(x)\right)^{p} d x\right]^{q / p} \\
& \leq c\left[\int_{\mathbb{R}} w_{1}(x) \sum_{j \in \mathbb{Z}}\left(\int_{|y|<x_{j}} w_{2}^{1-p^{\prime}}(y) d y\right)^{1-p}\left(\sum_{k=-\infty}^{j} \int_{E_{k}} f(x, \tau) d \tau\right)^{p} d x\right]^{q / p} .
\end{aligned}
$$

On the other hand, (3.9) yields

$$
\begin{aligned}
& \sum_{k=n}^{+\infty}\left(\int_{|\tau|<x_{k}} w_{2}^{1-p^{\prime}}(\tau) d \tau\right)^{1-p}\left(\sum_{k=-\infty}^{n} \int_{x_{k}<|\tau|<x_{k+1}} w_{2}^{1-p^{\prime}}(\tau) d \tau\right)^{p-1} \\
= & {\left[\sum_{k=n}^{+\infty}\left(\int_{|\tau|<x_{k}} w_{2}^{1-p^{\prime}}(\tau) d \tau\right)^{1-p}\right]\left(\int_{|\tau|<x_{n+1}} w_{2}^{1-p^{\prime}}(y) d y\right)^{p-1} } \\
= & \left(\sum_{k=n}^{+\infty} 2^{k(1-p)}\right) 2^{(n+1)(p-1)}=c<\infty
\end{aligned}
$$

for all $n \in \mathbb{Z}$. Hence, by Lemma 3.4 and Hölder's inequality with respect to the integral $\int_{x_{j}<|\tau|<x_{j+1}} f(x, \tau) d \tau$ we conclude that

$$
\begin{aligned}
I & \leq c\left[\int_{\mathbb{R}} w_{1}(x) \sum_{j \in \mathbb{Z}}\left(\int_{x_{j}<|y|<x_{j+1}} w_{2}^{1-p^{\prime}}(y) d y\right)^{1-p}\left(\int_{x_{j}<|\tau|<x_{j+1}} f(x, \tau) d \tau\right)^{p} d x\right]^{q / p} \\
& \leq c\left[\int_{\mathbb{R}} w_{1}(x) \sum_{j \in \mathbb{Z}}\left(\int_{x_{j}<|\tau|<x_{j+1}} w_{2}(\tau) f^{p}(x, \tau) d \tau\right) d x\right]^{q / p}=c D^{q}\|f\|_{L_{w}^{p}\left(\mathbb{R}^{2}\right)}^{q} .
\end{aligned}
$$

If $S<\infty$, then without loss of generality we can assume that $S=1$. In this case we choose the sequence $\left\{x_{k}\right\}_{k=-\infty}^{0}$ for which (3.9) holds for all $k \leq 0$. Arguing as in the case $S=\infty$ and using Lemma 3.5 instead of Lemma 3.4, we obtain the desired result. For necessity we put the functions

$$
\begin{aligned}
& f(x, y)=\chi_{\{x:|x-a|>r\}}(x) \chi_{\{y:|y|>t\}}(y) w^{1-p^{\prime}}(x, y)|x-a|^{\left(p^{\prime}-1\right)(\alpha-1)}, \\
& f(x, y)=\chi_{\{x:|x-a|<r\}}(x) \chi_{\{y:|y|>t\}}(y) w^{1-p^{\prime}}(x, y)
\end{aligned}
$$


$(a \in \mathbb{R} ; r, t>0)$ in the two-weight inequality and we are done.

Proof of Theorem 2.8. Let $f \geq 0$. Represent $I_{\alpha} J_{\beta}$ as follows:

$$
\begin{aligned}
I_{\alpha} J_{\beta} f(x, y)= & \int_{\mathbb{R}} \int_{|\tau|<|y| / 2} \frac{f(t, \tau)}{|x-t|^{1-\alpha}|\tau-y|^{1-\beta}} d t d \tau \\
& +\int_{\mathbb{R}} \int_{|y| / 2<|\tau|<2|y|} \frac{f(t, \tau)}{|x-t|^{1-\alpha}|\tau-y|^{1-\beta}} d t d \tau \\
:= & S_{\alpha, \beta}^{(1)} f(x, y)+S_{\alpha, \beta}^{(2)} f(x, y) .
\end{aligned}
$$

We have

$$
\left\|I_{\alpha} J_{\beta} f\right\|_{L_{v}^{q}\left(\mathbb{R}^{2}\right)}^{q} \leq c\left(\left\|S_{\alpha, \beta}^{(1)} f\right\|_{L_{v}^{q}\left(\mathbb{R}^{2}\right)}^{q}+\left\|S_{\alpha, \beta}^{(2)} f\right\|_{L_{v}^{q}\left(\mathbb{R}^{2}\right)}^{q}\right):=c\left(S^{(1)}+S^{(2)}\right) .
$$

If $|\tau|<|y| / 2$, then $|y-\tau|^{\beta-1} \leq c_{\beta}|y|^{\beta-1}$. Therefore, due to Proposition 3.3 we find that

$$
\begin{aligned}
S^{(1)} & \leq c \iint_{\mathbb{R}^{2}} v(x, y)|y|^{q(\beta-1)}\left(\int_{\mathbb{R}} \int_{|\tau|<|y| / 2} \frac{f(t, \tau)}{|x-t|^{1-\alpha}} d t d \tau\right)^{q} d x d y \\
& \leq c\left(\iint_{\mathbb{R}^{2}}(f(x, y))^{p} u(x) d x d y\right)^{q / p} .
\end{aligned}
$$

Here we have used the inequality

$$
\max \left\{B_{5}^{\prime}, B_{6}^{\prime}\right\} \leq c \max \left\{B_{5}, B_{6}\right\}
$$

where

$$
\begin{aligned}
& B_{5}^{\prime} \equiv \sup _{a \in \mathbb{R} ; r>0 ; s>0} s^{1 / p^{\prime}}\left(\int_{|x-a|>r} \int_{|y|>s} \frac{v(x, y)}{|x-a|^{(1-\alpha) q}|y|^{(1-\beta) q}} d x d y\right)^{1 / q} \\
& \times\left(\int_{|x-a|<r} u^{1-p^{\prime}}(x) d x\right)^{1 / p^{\prime}} \\
& B_{6}^{\prime} \equiv \sup _{a \in \mathbb{R} ; r>0 ; s>0} s^{1 / p^{\prime}}\left(\int_{|x-a|<r} \int_{|y|>s} \frac{v(x, y)}{|y|^{(1-\beta) q}} d x d y\right)^{1 / q} \\
& \times\left(\int_{|x-a|>r} \frac{u^{1-p^{\prime}}(x)}{|x-a|^{(1-\alpha) p^{\prime}}} d x\right)^{1 / p^{\prime}} .
\end{aligned}
$$

To check the latter inequality we take $r>0$ and $s>0$. Then $s \in\left[2^{n}, 2^{n+1}\right)$ for some $n \in \mathbb{Z}$. Hence

$$
\begin{aligned}
& s^{q / p^{\prime}}\left(\int_{|x-a|<r} u^{1-p^{\prime}}(x) d x\right)^{q / p^{\prime}} \int_{|x-a|>r} \int_{|y|>s} \frac{v(x, y)}{|x-a|^{(1-\alpha) q}|y|^{(1-\beta) q}} d x d y \\
\leq & c 2^{n q / p^{\prime}}\left(\int_{|x-a|<r} u^{1-p^{\prime}}(x) d x\right)^{q / p^{\prime}} \int_{|x-a|>r} \int_{|y|>2^{n}} \frac{v(x, y)}{|x-a|^{(1-\alpha) q}|y|^{(1-\beta) q}} d x d y
\end{aligned}
$$




$$
\begin{aligned}
= & c 2^{n q / p^{\prime}}\left(\int_{|x-a|<r} u^{1-p^{\prime}}(x) d x\right)^{q / p^{\prime}} \\
& \times \sum_{k=n}^{\infty} 2^{k(\beta-1) q} \int_{|x-a|>r} \int_{2^{k}<|y|<2^{k+1}} \frac{v(x, y)}{|x-a|^{(1-\alpha) q}} d x d y \\
\leq & c B_{5}^{q} 2^{n q / p^{\prime}} \sum_{k=n}^{\infty} 2^{-k q / p^{\prime}}=c B_{5}^{q} .
\end{aligned}
$$

Hence $B_{5}^{\prime} \leq c B_{5}$. Analogously it can be proved that $B_{6}^{\prime} \leq c B_{6}$.

Further, Hölder's inequality, Theorem A and simple calculations yield

$$
\begin{aligned}
& S^{(2)} \leq \iint_{\mathbb{R}^{2}} v(x, y)\left(\int_{\mathbb{R}}|x-t|^{\alpha-1}\left(\int_{|y| / 2<|\tau|<2|y|} f^{p}(t, \tau) d \tau\right)^{1 / p}\right. \\
& \left.\times\left(\int_{|y| / 2<|\tau|<2|y|}|y-\tau|^{(\beta-1) p^{\prime}} d \tau\right)^{1 / p^{\prime}} d t\right)^{q} d x d y \\
& =c \sum_{j \in \mathbb{Z}} \int_{\mathbb{R}} \int_{2^{j}<|y|<2^{j+1}} v(x, y)|y|^{q(\beta-1 / p)} \\
& \times\left(\int_{\mathbb{R}}|x-t|^{\alpha-1}\left(\int_{|y| / 2<|\tau|<2|y|} f^{p}(t, \tau) d \tau\right)^{1 / p} d t\right)^{q} d x \\
& \leq c \sum_{j \in \mathbb{Z}} \int_{\mathbb{R}}\left(\int_{2^{j}<|y|<2^{j+1}} v(x, y)|y|^{q(\beta-1 / p)} d y\right) \\
& \times\left(\int_{\mathbb{R}}|x-t|^{\alpha-1}\left(\int_{2^{j-1}<|\tau|<2^{j+2}} f^{p}(t, \tau) d \tau\right)^{1 / p} d t\right)^{q} d x \\
& \leq c\left(\max \left\{B_{5}, B_{6}\right\}\right)^{q} \sum_{j \in \mathbb{Z}}\left(\int_{\mathbb{R}} u(x) \int_{2^{j-1}<|x|<2^{j+2}} f^{p}(x, y) d x d y\right)^{q / p} \\
& \leq c\left(\iint_{\mathbb{R}^{2}} f^{p}(x, y) u(x) d x d y\right)^{q / p} .
\end{aligned}
$$

Necessity follows in the same manner as in the proof of Theorem 2.1 has been obtained.

Theorem 2.10 can be proved easier than the latter statement using the Hardy inequality (see, e.g., [19]):

$$
\int_{\mathbb{R}}(H f(x))^{p}|x|^{-p} d x \leq c \int_{\mathbb{R}}(f(x))^{p} d x .
$$

Indeed, let us first show sufficiency. Keeping the notation from the proof of the previous theorem, Theorem B and inequality (3.10) we will derive the following chain of inequalities:

$$
\left\|S_{\alpha, \beta}^{(1)} f\right\|_{L_{v}^{p}\left(\mathbb{R}^{2}\right)}^{p}
$$




$$
\begin{aligned}
& \leq c \int_{\mathbb{R}} \int_{\mathbb{R}} v(x, y)|y|^{(\beta-1) p}\left(\int_{\mathbb{R}} \int_{|\tau|<|y| / 2} \frac{f(t, \tau)}{|x-t|^{1-\alpha}} d t d \tau\right)^{p} d x d y \\
& =c \sum_{j \in \mathbb{Z}} \int_{\mathbb{R}} \int_{2^{j}<|y|<2^{j+1}} v(x, y)|y|^{(\beta-1) p}\left(\int_{\mathbb{R}} \int_{|\tau|<|y| / 2} \frac{f(t, \tau)}{|x-t|^{1-\alpha}} d t d \tau\right)^{p} d x d y \\
& \leq c \sum_{j \in \mathbb{Z}} 2^{j(1-p)} \int_{\mathbb{R}} \mathcal{V}_{j}(x)\left(\int_{\mathbb{R}}|x-t|^{\alpha-1}\left(\int_{|\tau|<2^{j}} f(t, \tau) d \tau\right) d t\right)^{p} d x \\
& \leq \sum_{j \in \mathbb{Z}} 2^{j(1-p)} \int_{\mathbb{R}}\left(\int_{|\tau|<2^{j}} f(t, \tau) d \tau\right)^{p} d t \\
& =c \int_{\mathbb{R}} \sum_{j} 2^{j(1-p)}\left(\int_{|\tau|<2^{j}} f(t, \tau) d \tau\right)^{p} d t \\
& \leq c \int_{\mathbb{R}}\left(\sum_{j} \int_{2^{j}<|x|<2^{j+1}}|x|^{-p}\left(\int_{|\tau|<|x|} f(t, \tau) d \tau\right)^{p} d x\right)^{p} d t \\
& =c \int_{\mathbb{R}}\left(\int_{\mathbb{R}}|x|^{-p}\left(\int_{|\tau|<|x|} f(t, \tau) d \tau\right)^{d x} d t \leq c \int_{\mathbb{R}} \int_{\mathbb{R}} f^{p}(t, \tau) d t d \tau .\right.
\end{aligned}
$$

Using again Theorem B we find that

$$
\begin{aligned}
& \left\|S_{\alpha, \beta}^{(2)} f\right\|_{L_{v}^{p}\left(\mathbb{R}^{2}\right)}^{p} \\
\leq & c \int_{\mathbb{R}} \int_{\mathbb{R}} v(x, y)\left(\int_{\mathbb{R}}|x-t|^{\alpha-1}\left(\int_{|y| / 2<|\tau|<|y|} f^{p}(t, \tau) d \tau\right)^{1 / p}\right. \\
& \left.\times\left(\int_{|y| / 2<|\tau|<|y|}|y-\tau|^{(\beta-1) p^{\prime}} d \tau\right)^{1 / p^{\prime}} d t\right)^{p} d x d y \\
\leq & c \sum_{j} \int_{\mathbb{R}} \mathcal{V}_{j}(x)\left(\int_{\mathbb{R}}|x-t|^{\alpha-1}\left(\int_{2^{j-1}<|\tau|<2^{j+1}} f^{p}(t, \tau) d \tau\right)^{1 / p} d t\right)^{p} d x d y \\
\leq & c \sum_{j} \int_{\mathbb{R}} \int_{2^{j-1}<|\tau|<2^{j+1}} f^{p}(t, \tau) d t d \tau \leq c\|f\|_{L^{p}\left(\mathbb{R}^{2}\right)}^{p} \cdot
\end{aligned}
$$

Necessity. To prove necessity we observe that the boundedness of the operator $I_{\alpha} J_{\beta}$ from $L^{p}\left(\mathbb{R}^{2}\right)$ to $L_{v}^{p}\left(\mathbb{R}^{2}\right)$ implies the inequality

$$
\int_{\mathbb{R}} \mathcal{V}_{j}(x)\left(\int_{\mathbb{R}}|x-t|^{\alpha-1} g(t) d t\right)^{p} d x \leq c\|g\|_{L^{p}(\mathbb{R})}^{p}
$$

for all non-negative functions $g$ defined on $\mathbb{R}$ and all $j \in \mathbb{Z}$. This is possible if we put the functions of the form $f_{j}(x, y)=g(x) \chi_{\left\{y: 2^{j-2}<|y|<2^{j-1}\right\}}(y)$ in the inequality

$$
\left\|I_{\alpha} J_{\beta} f\right\|_{L_{v}^{p}\left(\mathbb{R}^{2}\right)} \leq c\|f\|_{L^{p}\left(\mathbb{R}^{2}\right)} .
$$


Further, by Theorem B we conclude that $I_{\alpha} \mathcal{V}_{j} \in L_{\text {loc }}^{p^{\prime}}(\mathbb{R})$ for all $j \in \mathbb{Z}$ and

$$
\sup _{x \in \mathbb{R} ; j \in \mathbb{Z}}\left(\frac{I_{\alpha}\left[I_{\alpha} \mathcal{V}_{j}\right]^{p^{\prime}}(x)}{I_{\alpha} \mathcal{V}_{j}(x)}\right)^{1 / p^{\prime}}<\infty
$$

Proof of Theorem 2.11. First we prove the statement for the dyadic strong fractional maximal function $N_{\alpha, \beta} f(x, y)$. Suppose that $f \geq 0, f$ is bounded and has a compact support. Arguing as in the proof of Theorem 2.2 for given $(x, y) \in \mathbb{R}^{2}$ we take dyadic intervals $I(x)$ and $J(y)(x \in I(x), y \in J(y))$ so that (3.6) holds. Using the notation of the proof of Theorem 2.2 we find that

$$
\begin{aligned}
I & :=\iint_{\mathbb{R}^{2}}\left(N_{\alpha, \beta} f\right)^{q}(x, y) v(x, y) d x d y \leq 2^{q} \sum_{I, J \in \mathcal{D}} \bar{V}_{I, J} \bar{F}_{I, J}^{q} \\
& =2^{q} \sum_{I, J \in \mathcal{D}}\left(\int_{I} \int_{J} f(x, y)\left(\int_{I} \int_{J} v(t, \tau) d t d \tau\right)^{1 / q} d x d y\right)^{q}|I|^{(\alpha-1) q}|J|^{(\beta-1) q} \\
& =2^{q} \sum_{I, J \in \mathcal{D}} \frac{1}{(|I||J|)^{q / p^{\prime}}}\left(\int_{I} \int_{J} f(x, y)\left(|I|^{q(\alpha-1 / p)}|J|^{q(\beta-1 / p)} \int_{I} \int_{J} v(t, \tau) d t d \tau\right)^{1 / q} d x d y\right)^{q} \\
& \leq 2^{q} \sum_{I, J \in \mathcal{D}}|I|^{-q / p^{\prime}}|J|^{-q / p^{\prime}}\left(\int_{I} \int_{J} f(x, y)\left(\widetilde{N}_{\alpha, \beta} v\right)^{1 / q}(x, y) d x d y\right)^{q},
\end{aligned}
$$

where

$$
\tilde{N}_{\alpha, \beta} v(x, y)=\sup _{I \ni x ; J \ni y ; I, J \in \mathcal{D}}|I|^{q(\alpha-1 / p)}|J|^{q(\beta-1 / p)} \int_{I} \int_{J} v(t, \tau) d t d \tau .
$$

Applying Corollary A twice with $\rho(x) \equiv 1$ and Minkowski's inequality we obtain

$$
\begin{aligned}
I & \leq c \sum_{I \in \mathcal{D}}|I|^{-q / p^{\prime}}\left(\int_{\mathbb{R}}\left(\int_{I} f(x, y)\left(\widetilde{N}_{\alpha, \beta} v\right)^{1 / q}(x, y) d x\right)^{p} d y\right)^{q / p} \\
& \leq c \sum_{I \in \mathcal{D}}|I|^{-q / p^{\prime}}\left(\int_{I}\left(\int_{\mathbb{R}} f^{p}(x, y)\left(\widetilde{N}_{\alpha, \beta} v\right)^{p / q}(x, y) d y\right)^{1 / p} d x\right)^{q} \\
& \leq c\left(\int_{\mathbb{R}} \int_{\mathbb{R}} f^{p}(x, y)\left(\tilde{N}_{\alpha, \beta} v\right)^{p / q}(x, y) d x d y\right)^{q / p} .
\end{aligned}
$$

Let us now pass to $M_{\alpha, \beta}$. Keeping the notation of Lemma 3.1 we have

$$
\begin{aligned}
D_{t, \tau} & :=\iint_{\mathbb{R}^{2}}\left(S_{t, \tau}(x, y)\right)^{q} v(x, y) d x d y \\
& =\iint_{\mathbb{R}^{2}}\left(\sup _{I-t \ni x ; J-t \ni y ; I, J \in \mathcal{D}}|I|^{\alpha-1}|J|^{\beta-1} \int_{I-t} \int_{J-\tau} f(s, \varepsilon) d s d \varepsilon\right)^{q} v(x, y) d x d y \\
& =\iint_{\mathbb{R}^{2}}\left(\sup _{I \ni x ; J \ni y ; I, J \in \mathcal{D}}|I|^{\alpha-1}|J|^{\beta-1} \int_{I} \int_{J} f(s-t, \varepsilon-\tau) d s d \varepsilon\right)^{q} v(x-t, y-\tau) d x d y
\end{aligned}
$$




$$
\begin{aligned}
& =\iint_{\mathbb{R}^{2}}(N f(\cdot-t, \cdot-\tau))^{q}(x, y) v(x-t, y-\tau) d x d y \\
& \leq c\left(\iint_{\mathbb{R}^{2}}(f(x-t, y-\tau))^{p}\left(\widetilde{N}_{\alpha, \beta} v(\cdot-t, \cdot-\tau)\right)^{p / q}(x, y) d x d y\right)^{q / p} \\
& =c\left(\int \int _ { \mathbb { R } ^ { 2 } } ( f ( x - t , y - \tau ) ) ^ { p } \left(\sup _{I \ni x ; J \ni y ; I, J \in \mathcal{D}}|I|^{q(\alpha-1 / p)}|J|^{q(\beta-1 / p)}\right.\right. \\
& \left.\left.\times \int_{I} \int_{J} v(s-t, \varepsilon-\tau) d s d \varepsilon\right)^{p / q} d x d y\right)^{q / p} \\
& =c\left(\int \int _ { \mathbb { R } ^ { 2 } } ( f ( x - t , y - \tau ) ) ^ { p } \left(\sup _{I \ni x ; J \ni y ; I, J \in \mathcal{D}}|I-t|^{q(\alpha-1 / p)}|J-\tau|^{q(\beta-1 / p)}\right.\right. \\
& \left.\left.\times \int_{I-t} \int_{J-\tau} v(s, \varepsilon) d s d \varepsilon\right)^{p / q} d x d y\right)^{q / p} \\
& \leq c\left(\iint_{\mathbb{R}^{2}} f^{p}(x, y)\left(\tilde{N}_{\alpha, \beta} v(\cdot-t, \cdot-\tau)\right)^{p / q}(x, y) d x d y\right)^{q / p} \\
& \leq c\left(\iint_{\mathbb{R}^{2}}(f(x, y))^{p}\left(\widetilde{M}_{\alpha, \beta} v\right)^{p / q}(x, y) d x d y\right)^{q / p} .
\end{aligned}
$$

Taking into account the proof of the latter theorem we can formulate the next statement for the classical fractional maximal function $M_{\gamma}$.

Lemma 3.6. Let $1<p<q<\infty$. Suppose that $\rho$ is an one-dimensional weight. Assume that $0<\gamma<1 / p$. Then the inequality

$$
\left(\int_{\mathbb{R}}\left(M_{\gamma} f(x)\right)^{q} \rho(x) d x\right)^{1 / q} \leq c\left(\int_{\mathbb{R}}|f(x)|^{p}\left(\widetilde{M}_{\gamma} \rho\right)^{p / q}(x) d x\right)^{1 / p}
$$

holds with a positive constant $c$ independent of $f$ and $\rho$, where

$$
\left(\widetilde{M}_{\gamma} \rho\right)^{p / q}(x)=\sup _{I \ni x}|I|^{q(\gamma-1 / p)} \int_{I} \rho(x) d x .
$$

Proposition 3.2 and Lemma 3.6 yield:

Corollary 3.7. Let $1<p<q<\infty$ and let $\rho \in A_{\infty}(\mathbb{R})$. Suppose that $0<\gamma<$ $1 / p$. Then the inequality

$$
\left(\int_{\mathbb{R}}\left|I_{\gamma} f(x)\right|^{q} \rho(x) d x\right)^{1 / q} \leq c\left(\int_{\mathbb{R}}|f(x)|^{p}\left(\widetilde{M}_{\gamma} \rho\right)^{p / q}(x) d x\right)^{1 / p}
$$

holds with a positive constant $c$ independent of $f$.

Proof of Theorem 2.12. As before we assume that $f$ is non-negative bounded and has a compact support. Using the notation of the proof of Theorem 2.1 we have

$J:=\int_{\mathbb{R}} \int_{\mathbb{R}}\left(N_{\alpha} I_{\beta} f\right)^{q}(x, y) v(x, y) d x d y$ 


$$
\begin{aligned}
& \leq 2^{q} \sum_{I \in \mathcal{D}_{m}} \int_{\mathbb{R}} \bar{V}_{I}(y)\left[\int_{\mathbb{R}}|y-\tau|^{\beta-1} \bar{F}_{I}(\tau) d \tau\right]^{q} d y \\
& =2^{q} \sum_{I \in \mathcal{D}_{m}}|I|^{(\alpha-1) q} \int_{\mathbb{R}}\left(\int_{I} v(x, y) d x\right)\left(\int_{\mathbb{R}} \bar{F}_{I}(\tau)|y-\tau|^{\beta-1} d \tau\right)^{q} d y
\end{aligned}
$$

(by Corollary 3.7 and the fact $\int_{I} v(x, \cdot) d x \in A_{\infty}(\mathbb{R})$ with the constant independent of $I$ )

$\leq c \sum_{I \in \mathcal{D}_{m}}|I|^{(\alpha-1) q}\left[\int_{\mathbb{R}}\left(\int_{I} f(t, y) d t\right)^{p}\left[\widetilde{M}_{\beta}\left(\int_{I} v(x, \cdot) d x\right)\right]^{p / q}(y) d y\right]^{q / p}$

(generalized Hölder's inequality)

$$
\begin{aligned}
& \leq c \sum_{I \in \mathcal{D}_{m}}|I|^{(\alpha-1) q}\left(\int_{I}\left[\int_{\mathbb{R}} f^{p}(t, y)\left[\widetilde{M}_{\beta}\left(\int_{I} v(x, \cdot) d x\right)\right]^{p / q}(y) d y\right]^{1 / p} d t\right)^{q} \\
& =c \sum_{I \in \mathcal{D}_{m}} \frac{1}{|I|^{(1-\alpha) q}}\left(\int_{I}\left[\int_{\mathbb{R}} f^{p}(t, y)\left[\sup _{J \ni y ; J \in \mathcal{D}_{m}} \frac{1}{|J|^{(1 / p-\beta) q}} \int_{I} \int_{J} v(x, \tau) d x d \tau\right]^{p / q} d y\right]^{1 / p} d t\right)^{q} \\
& =c \sum_{I \in \mathcal{D}_{m}} \frac{1}{|I|^{q / p^{\prime}}}\left(\int_{I}\left[\int_{\mathbb{R}} f^{p}(t, y)\left[\sup _{J \ni y ; J \in \mathcal{D}_{m}} \frac{1}{|I|^{(1 / p-\alpha) q|J|^{(1 / p-\beta) q}}} \int_{I} \int_{J} v(x, \tau) d x d \tau\right]^{\frac{p}{q}} d y\right]^{\frac{1}{p}} d t\right)^{q}
\end{aligned}
$$

(by Corollary A with $\rho(x) \equiv 1$ )

$\leq c\left(\iint_{\mathbb{R}^{2}}(f(x, y))^{p}\left(\widetilde{M}_{\alpha, \beta} v\right)^{p / q}(x, y) d x d y\right)^{q / p}$.

Arguments similar to that of the proof of the previous theorem completes the proof.

Proof of Theorem 2.13. We have

$$
\begin{aligned}
& \iint_{\mathbb{R}^{2}}\left|I_{\alpha, \beta} f(x, y)\right|^{q} v(x, y) d x d y \\
\leq & \int_{\mathbb{R}}\left(\int_{\mathbb{R}} v(x, y)\left(\int_{\mathbb{R}^{2}}|x-t|^{\alpha-1}\left(\int_{\mathbb{R}} \frac{|f(t, \tau)|}{|y-\tau|^{1-\beta}} d \tau\right) d t\right)^{q} d y\right) d x
\end{aligned}
$$

( by Proposition 3.2 since $v \in A_{\infty}^{(x)}(\mathbb{R})$ )

$$
\leq c \iint_{\mathbb{R}^{2}}\left(M_{\alpha} I_{\beta} f(x, y)\right)^{q} v(x, y) d x d y
$$

(by Theorem 2.12 since $v \in A_{\infty}^{(y)}(\mathbb{R})$ )

$$
\leq c\left(\iint_{\mathbb{R}^{2}}|f(x, y)|^{p}\left(\widetilde{M}_{\alpha, \beta} v\right)^{p / q}(x, y) d x d y\right)^{q / p} \text {. }
$$

Remark 3.8. It is known that $\left(M_{\gamma} \rho\right)^{\lambda}$, where $\rho$ is a one-dimensional function and $0<\lambda<1$, belongs to the Muckenhoupt's class $A_{1}$ (see, e.g., [36]), i.e., 
there exists a positive constant $c$ such that for all intervals $I \subset \mathbb{R}$ with finite length the inequality

$$
1 /|I| \int_{I}\left(M_{\gamma} \rho\right)^{\lambda} \leq c \underset{I}{\operatorname{ess} \inf }\left(M_{\gamma} \rho\right)^{\lambda}
$$

holds. Let now $0<\alpha, \beta<1 / p$.

From the latter fact and the inequalities

$$
\begin{aligned}
\left(\widetilde{M}_{\alpha, \beta} v\right)(x, y) & \leq \sup _{I \ni x} \frac{1}{|I|^{q(1 / p-\alpha)}} \int_{I}\left(\sup _{J \ni y} \frac{1}{|J|^{q(1 / p-\beta)}} \int_{J} v(t, \tau) d \tau\right) d t \\
& :=\left(\widetilde{M}_{\alpha} v_{1}(\cdot, y)\right)(x) ; \\
\left(\widetilde{M}_{\alpha, \beta} v\right)(x, y) & \leq \sup _{J \ni y} \frac{1}{|J|^{q(1 / p-\beta)}} \int_{J}\left(\sup _{I \ni x} \frac{1}{|I|^{q(1 / p-\alpha)}} \int_{I} v(t, \tau) d t\right) d \tau \\
& :=\left(\widetilde{M}_{\beta} v_{2}(x, \cdot)\right)(y),
\end{aligned}
$$

where

$$
v_{1}(t, y):=\sup _{J \ni y}|J|^{q(\beta-1 / p)} \int_{J} v(t, \tau) d \tau ; \quad v_{2}(x, \tau):=\sup _{I \ni x}|I|^{q(\alpha-1 / p)} \int_{I} v(t, \tau) d t,
$$

it follows that $\left(\widetilde{M}_{\alpha, \beta} v\right)^{\lambda}(\cdot, y) \in A_{1}(\mathbb{R})$ for every $y \in \mathbb{R}$ and $\left(\widetilde{M}_{\alpha, \beta} v\right)^{\lambda}(x, \cdot) \in$ $A_{1}(\mathbb{R})$ for every $x \in \mathbb{R}$.

Remark 3.9. An analysis of proofs of the main results enables us to conclude that all statements concerning $M_{\alpha, \beta}, I_{\alpha, \beta}$ and $I_{\alpha} J_{\beta}$ hold also for the following operators defined on $\mathbb{R}^{n} \times \mathbb{R}^{m}(n, m>1)$ :

$$
\begin{aligned}
&\left(M_{\alpha, \beta}^{(n, m)} f\right)(x, y)=\sup _{B_{n} \times B_{m} \ni(x, y)} \frac{1}{\left|B_{n}\right|^{1-\alpha / n}\left|B_{m}\right|^{1-\beta / m}} \int_{B_{n}} \int_{B_{m}}|f(z, s)| d z d s ; \\
&\left(I_{\alpha, \beta}^{(n, m)} f\right)(x, y)=\int_{\mathbb{R}^{n}} \int_{\mathbb{R}^{m}} \frac{f(z, s)}{|x-z|^{n-\alpha}|y-s|^{m-\beta}} d z d s, \quad x \in \mathbb{R}^{n}, y \in \mathbb{R}^{m} ; \\
&\left(I_{\alpha}^{(n)} J_{\beta}^{(m)} f\right)(x, y)=\int_{\mathbb{R}^{n}} \int_{\left\{\tau \in \mathbb{R}^{m}:|\tau|<2|y|\right\}} \frac{f(t, \tau)}{|x-t|^{n-\alpha}|y-\tau|^{m-\beta}} d t d \tau, \\
& x \in \mathbb{R}^{n}, y \in \mathbb{R}^{m}
\end{aligned}
$$

respectively, where $0<\alpha<n, 0<\beta<m$ and $B_{d}$ is a ball in $\mathbb{R}^{d}$.

Acknowledgement. The authors express their gratitude to the referee for remarks and suggestions.

\section{References}

[1] D. R. Adams, A trace inequality for generalized potentials, Studia Math. 48 (1973), 99-105.

[2] _ Lectures on $L^{p}$-potential theory, Umea Univ. Reports, No.2, 1981.

[3] D. R. Adams and L. I. Hedberg, Function Spaces and Potential Theory, Springer- Verlag, Berlin, 1996. 
[4] J. S. Bradley, Hardy inequalities with mixed norms, Canad. Math. Bull. 21 (1978), no. $4,405-408$.

[5] L. Carleson, An interpolation problem for bounded analytic functions, Amer. J. Math. 80 (1958), 921-930.

[6] S. Chanillo, D. K. Watson, and R. L. Wheeden, Some integral and maximal operators related to starlike sets, Studia Math. 107 (1993), no. 3, 223-255.

[7] R. R. Coifman and C. Fefferman, Weighted norm inequalities for maximal functions and singular integrals, Studia Math. 51 (1974), 241-250.

[8] E. M. Dynkin and B. P. Osilenker, Weighted estimates for singular integrals and their applications, Mathematical analysis, Vol. 21, 42-129, Itogi Nauki i Tekhniki, Akad. Nauk SSSR, Vsesoyuz. Inst. Nauchn. i Tekhn. Inform., Moscow, 1983.

[9] D. E. Edmunds, V. Kokilashvili, and A. Meskhi, On Fourier multipliers in weighted Triebel-Lizorkin spaces, J. Inequal. Appl. 7 (2002), no. 4, 555-591.

[10] , Bounded and Compact Integral Operators, Mathematics and its Applications, Vol. 543, Kluwer Academic Publishers, Dordrecht, Boston, London, 2002.

[11] R. Fefferman, Multiparameter Fourier analysis, Beijing lectures in harmonic analysis (Beijing, 1984), 47-130, Ann. of Math. Stud., 112, Princeton Univ. Press, Princeton, NJ, 1986.

[12] C. Fefferman and E. Stein, Some maximal inequalities, Amer. J. Math. 93 (1971), 107115.

[13] M. Gabidzashvili, Weighted inequalities for anisotropic potentials, Trudy Tbiliss. Mat. Inst. Razmadze Akad. Nauk Gruzin. SSR 82 (1986), 25-36.

[14] M. Gabidzashvili, I. Genebashvili, and V. Kokilashvili, Two-weight inequalities for generalized potentials, Trudy Mat. Inst. Steklov. 194 (1992), Issled. po Teor. Differ. Funktsii Mnogikh Peremen. i ee Prilozh. 14, 89-96; translation in Proc. Steklov Inst. Math. 194 (1993), no. 4, 91-99.

[15] M. Gabidzashvili and V. Kokilashvili, Two weight weak type inequalities for fractional type integrals, Preprint, No. 45, Mathematical Institute Czech Acad. Sci., Prague, 1989.

[16] J. García-Cuerva and J. L. Rubio de Francia, Weighted Norm Inequalities and Related Topics, North-Holland Mathematics Studies, 116, Mathematical Notes, 104, NorthHolland Publishing Co., Amsterdam, 1985.

[17] I. Genebashvili, A. Gogatishvili, V. Kokilashvili, and M. Krbec, Weight Theory for Integral Transforms on Spaces of Homogeneous Type, Pitman Monographs and Surveys in Pure and Applied Mathematics, 92. Longman, Harlow, 1998.

[18] A. Gogatishvili and V. Kokilashvili, Criteria of strong type two-weighted inequalities for fractional maximal functions, Georgian Math. J. 3 (1996), no. 5, 423-446.

[19] G. H. Hardy, J. E. Littlewood, and G. Polya, Inequalities, Cambridge Univ. Press, 1934.

[20] L. Hörmander, $L^{p}$ estimates for (pluri-) subharmonic functions, Math. Scand. 20 (1967), 65-78.

[21] R. Hunt, B. Muckenhoupt, and R. L. Wheeden, Weighted norm inequalities for the conjugate function and Hilbert transform, Trans. Amer. Math. Soc. 176 (1973), 227251.

[22] V. M. Kokilashvili, Weighted Lizorkin-Triebel spaces. Singular integrals, multipliers, imbedding theorems, Trudy Mat. Inst. Steklov. 161 (1983), 125-149.

[23] _ Bisingular integral operators in weighted spaces, Soobshch. Akad. Nauk Gruzin. SSR 101 (1981), no. 2, 289-292.

[24] On Hardy's inequalities in weighted spaces, Soobshch. Akad. Nauk Gruzin. SSR 96 (1979), no. 1, 37-40.

[25] V. Kokilashvili and M. Krbec, Weighted Inequalities in Lorentz and Orlicz Spaces, World Scientific Publishing Co., Inc., River Edge, NJ, 1991.

[26] V. Kokilashvili and A. Meskhi, On one-sided potentials with multiple kernels, Integral Transforms Spec. Funct. 16 (2005), no. 8, 669-683. 
[27] _ On two-weight estimates for strong fractional maximal functions and potentials with multiple kernels, Proc. A. Razmadze Math. Inst. 137 (2005), 135-140.

[28] _ On a trace inequality for one-sided potentials with multiple kernels, Fract. Calc. Appl. Anal. 6 (2003), no. 4, 461-472.

[29] _ Two-weighted criteria for integral transforms with multiple kernels, Approximation and probability, 119-140, Banach Center Publ., 72, Polish Acad. Sci., Warsaw, 2006.

[30] V. G. Maz'ya, Sobolev Spaces, Springer, Berlin, 1985.

[31] V. G. Maz'ya and I. E. Verbitsky, Capacitary inequalities for fractional integrals, with applications to partial differential equations and Sobolev multipliers, Ark. Mat. 33 (1995), no. 1, 81-115.

[32] A. Meskhi, A note on two-weight inequalities for multiple Hardy-type operators, J. Funct. Spaces Appl. 3 (2005), no. 3, 223-237.

[33] B. Muckenhoupt, Hardy's inequality with weights, Studia Math. 44 (1972), 31-38.

[34] B. Muckenhoupt and R. L. Wheeden, Weighted norm inequalities for fractional integrals, Trans. Amer. Math. Soc. 192 (1974), 261-274.

[35] S. G. Samko, A. A. Kilbas, and O. I. Marichev, Fractional Integrals and Derivative, Theory and Applications, Gordon and Breach Sci. Publishers, 1993.

[36] E. T. Sawyer, Two weight norm inequalities for certain maximal and integral operators, Harmonic analysis (Minneapolis, Minn., 1981), pp. 102-127, Lecture Notes in Math., 908, Springer, Berlin-New York, 1982.

[37] _ Weighted norm inequalities for fractional maximal operators, in: Seminar on Harmonic Analysis,Montreal, Que., 1980, pp. 283-309, CMS Conf. Proc., 1, Amer. Math. Soc.Providence, R.I., 1981.

[38] _ A two weight weak type inequality for fractional integrals, Trans. Amer. Math. Soc. 281 (1984), no. 1, 339-345.

[39] _ Multipliers of Besov and power-weighted $L^{2}$ spaces, Indiana Univ. Math. J. 33 (1984), no. 3, 353-366.

[40] A characterization of two weight norm inequalities for fractional and Poisson integrals, Trans. Amer. Math. Soc. 308 (1988), no. 2, 533-545.

[41] E. T. Sawyer and R. L. Wheeden, Weighted inequalities for fractional integrals on Euclidean and homogeneous spaces, Amer. J. Math. 114 (1992), no. 4, 813-874.

[42] E. T. Sawyer, R. L. Wheeden, and S. Zhao, Weighted norm inequalities for operators of potential type and fractional maximal functions, Potential Anal. 5 (1996), no. 6, 523-580.

[43] J. O. Strömberg and A. Torchinsky, Weighted Hardy Spaces, Lecture Notes in Math., Vol. 1381, Springer Verlag, Berlin, 1989.

[44] K. Tachizawa, On weighted dyadic Carleson's inequalities, J. Inequal. Appl. 6 (2001), no. 4, 415-433.

[45] I. E. Verbitsky and R. L. Wheeden, Weighted norm inequalities for integral operators, Trans. Amer. Math. Soc. 350 (1998), no. 8, 3371-3391.

[46] R. L. Wheeden, A characterization of some weighted norm inequalities for the fractional maximal function, Studia Math. 107 (1993), no. 3, 257-272.

VAKHTANG KOKILASHVILI

A. Razmadze Mathematical Institute

1. M. Aleksidze Str.

0193 Tbilisi, Georgia

E-mail address: kokil@rmi.acnet.ge 
Alexander Meskhi

A. Razmadze Mathematical Institute

1. M. Aleksidze Str.

0193 TBilisi, Georgia

E-mail address: meskhi@rmi.acnet.ge 